\title{
NSUN5 is Upregulated and Positively Correlated with Translation in Human Cancers: A Bioinformatics-based Study
}

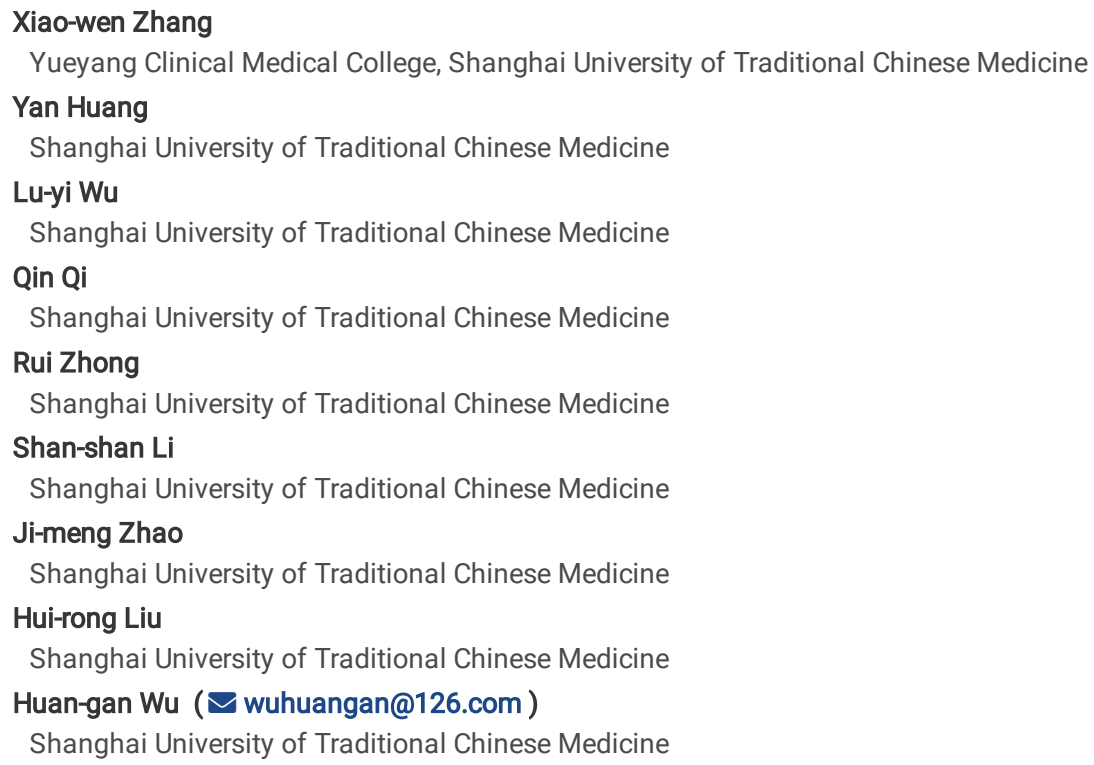




\section{Abstract}

The role of RNA $\mathrm{m}^{5} \mathrm{C}$ (5-methylcytosine) and RNA $\mathrm{m}^{5} \mathrm{C}$ methyltransferases (RCMTs, including NSUN1, NSUN2, NSUN3, NSUN4, NSUN5, NSUN6, NSUN7 and TRDMT1) in human cancers remains largely unknown. In this study, GEPIA2 was used to compare the expression of RCMTs in human cancers and that in associated normal tissues, and to analyze the prognosis value of NSUN5 expression. UALCAN was used to compare the methylation level of NSUN5 promoter in human cancers and that in associated normal tissues. LinkedOmics was used perform BPs (biological processes), CCs (cellular components), MFs (molecular functions) and KEGG pathways analyses of NSUN5-correlated genes in each cancer one by one. We found that six RCMTs (NSUN1-NSUN5 and TRDMT1), especially NSUN5, were generally upregulated in human cancers, that the hypomethylation of NSUN5 promoter may be responsible for its upregulation, and that overexpressed NSUN5 predicted poorer prognosis and was positively correlated with translation in human cancers. The function of NSUN5 in human cancers and its mechanism need to be validated by biological experiments.

\section{Introduction}

According to MODOMICS (https://iimcb.genesilico.pl/modomics/), there are at least 171 types of RNA modifications so far. ${ }^{[1]} \mathrm{m}^{6} \mathrm{~A}\left(\mathrm{~N}^{6}\right.$-methyladenosine) is the most pervasive RNA modification, and can affect growth and metastasis in multiple cancers (glioblastoma, lung cancer, hepatocellular carcinoma, et al) by influencing RNA biogenesis, splicing, degradation or translation. ${ }^{[2]}$ However, the role of other types of RNA modifications in human cancers is poorly studied.

In recent years, $\mathrm{m}^{5} \mathrm{C}$ (5-methylcytosine) has been found to be widespread in human rRNA (ribosomal RNA), tRNA (transfer RNA) and mRNA (messenger RNA). $[3,4]$ There are eight kinds of human RNA $m^{5} \mathrm{C}$ methyltransferases (RCMTs): NSUN1 (NOP2), NSUN2, NSUN3, NSUN4, NSUN5, NSUN6, NSUN7 and TRDMT1 (DNMT2). ${ }^{[5]}$ Only a small number of studies has reported the role of $\mathrm{m}^{5} \mathrm{C}$ and RCMTs in human cancers. For example, $\mathrm{m}^{5} \mathrm{C}$ and RCMTs (NSUN1, NSUN3 and TRDMT1) mediate chromatin organization and 5-azacytidine response and resistance in leukemia. ${ }^{[6]} \mathrm{m}^{5} \mathrm{C}$, induced by upregulated NSUN2, promotes pathogenesis of bladder cancer through stabilizing oncogene mRNAs. ${ }^{[7]} \mathrm{m}^{5} \mathrm{C}$ regulation and its functions in human cancers remains largely unknown and need further research.

In this study, using public databases, we analyzed the expression, prognostic value and possible function of NSUN5 (the most obviously upregulated RCMT) in multiple human cancers.

\section{Results}

\section{The expression of RCMTs, especially NSUN5, is generally upregulated in cancers}

GEPIA2 ${ }^{[8]}$ (http://gepia2.cancer-pku.cn/\#index) was used to explore the expression of RCMTs in cancers. As is shown in Table 1 and Fig. 1, the expression of RCMTs, except NSUN6 and NSUN7, is generally upregulated in cancers, compared to associated normal tissues respectively. In detail, NSUN1, NSUN2, NSUN3, NSUN4, NSUN5, NSUN6, NSUN7 and TRDMT1 were respectively upregulated in 23, 19, 18, 18, 25, 7, 12 and 13 types of cancers out of all the 31 types of cancers. Among them, the number of cancer types with upregulated NSUN5 was the most. (Table 1) NSUN5 was upregulated in ACC (Adrenocortical carcinoma), BLCA (Bladder Urothelial Carcinoma), BRCA (Breast invasive carcinoma), CESC (Cervical squamous cell carcinoma and endocervical adenocarcinoma), CHOL (Cholangiocarcinoma), COAD (Colon adenocarcinoma), DLBC (Lymphoid Neoplasm Diffuse Large B-cell Lymphoma), ESCA (Esophageal carcinoma), GBM (Glioblastoma multiforme), HNSC (Head and Neck squamous cell carcinoma), KICH (Kidney Chromophobe), KIRP (Kidney renal papillary cell carcinoma ), LIHC (Liver hepatocellular carcinoma), LUAD (Lung adenocarcinoma), LUSC (Lung squamous cell carcinoma), OV (Ovarian serous cystadenocarcinoma), PAAD (Pancreatic adenocarcinoma), PRAD (Prostate adenocarcinoma), READ (Rectum adenocarcinoma), SKCM (Skin Cutaneous Melanoma), STAD (Stomach adenocarcinoma), TGCT (Testicular Germ Cell Tumors), THYM (Thymoma), UCEC (Uterine Corpus Endometrial Carcinoma) and UCS (Uterine Carcinosarcoma), downregulated in KIRC (Kidney renal clear cell carcinoma), LAML (Acute Myeloid Leukemia) and THCA (Thyroid carcinoma), and not significantly changed in LGG (Brain Lower Grade Glioma), PCPG (Pheochromocytoma and Paraganglioma) and SARC (Sarcoma). (Fig. 1) Therefore, we chose NSUN5 for further study. 
The expression of RCMTs in 31 types of cancers according to GEPIA2.

\begin{tabular}{|c|c|c|c|c|c|c|c|c|}
\hline $\begin{array}{l}\text { RCMTs } \\
\text { Cancers }\end{array}$ & NSUN1 & NSUN2 & NSUN3 & NSUN4 & NSUN5 & NSUN6 & NSUN7 & TRDMT1 \\
\hline ACC & Down & NS & Down & Down & Up & Down & Down & Down \\
\hline BLCA & Up & Up & NS & NS & Up & NS & NS & Down \\
\hline BRCA & Up & Up & Up & Up & Up & Down & Up & Down \\
\hline CESC & Up & NS & NS & NS & Up & Down & NS & Down \\
\hline $\mathrm{CHOL}$ & Up & Up & Up & Up & Up & Down & Up & Up \\
\hline COAD & Up & Up & Up & Up & Up & Up & Up & Up \\
\hline DLBC & Up & Up & Up & Up & Up & Up & Down & Up \\
\hline ESCA & Up & Up & Up & Up & Up & NS & NS & Up \\
\hline GBM & Up & Up & Up & Up & Up & Down & Up & Up \\
\hline HNSC & Up & Up & Up & Up & Up & Up & Down & NS \\
\hline $\mathrm{KICH}$ & Down & Up & NS & Down & Up & Down & Up & Down \\
\hline KIRC & Up & Up & NS & NS & Down & NS & Down & NS \\
\hline KIRP & Up & Up & NS & Up & Up & Up & NS & NS \\
\hline LAML & Up & Down & Up & Down & Down & Up & Up & Up \\
\hline LGG & Up & Up & Up & Up & NS & Up & Up & Up \\
\hline LIHC & Up & NS & Up & Up & Up & Down & NS & Up \\
\hline LUAD & NS & Up & Up & Up & Up & Down & NS & Down \\
\hline LUSC & Up & Up & Up & Up & Up & Down & Down & Down \\
\hline OV & Up & Down & Up & Up & Up & Down & Up & Down \\
\hline PAAD & Up & Up & Up & Up & Up & NS & Down & Up \\
\hline PCPG & NS & NS & NS & Down & NS & NS & Down & NS \\
\hline PRAD & Down & Down & NS & NS & Up & Down & Up & NS \\
\hline READ & Up & Up & Up & Up & Up & NS & Up & Up \\
\hline SARC & Up & Up & NS & NS & NS & NS & NS & NS \\
\hline SKCM & Up & Down & Up & Up & Up & Down & Down & Up \\
\hline STAD & Up & Up & Up & Up & Up & Down & Down & Up \\
\hline TGCT & Up & NS & NS & Down & Up & Down & Down & Down \\
\hline THCA & Down & Down & Down & Down & Down & Down & Down & Down \\
\hline THYM & Up & Up & Up & Up & Up & Up & Down & Up \\
\hline UCEC & NS & Down & Down & NS & Up & Down & Up & Down \\
\hline UCS & NS & Down & Down & NS & Up & Down & Up & Down \\
\hline $\begin{array}{l}\text { Number of Cancer Types } \\
\text { (Up: NS: Down) }\end{array}$ & $23: 4: 4$ & $19: 5: 7$ & $18: 9: 4$ & $18: 7: 6$ & $25: 3: 3$ & $7: 7: 17$ & $12: 7: 12$ & $13: 6: 12$ \\
\hline
\end{tabular}

\section{The hypomethylation of NSUN5 promoter may be responsible for its upregulation in cancers}

To explore why NSUN5 was generally downregulated in cancers, we compared the methylation level of NSUN5 promoter in cancers and associated normal tissues using UALCAN ${ }^{[9]}$ (http://ualcan.path.uab.edu/). Among the 28 types of cancers with DNA methylation data, the methylation level of NSUN5 promoter was downregulated in BRCA, HNSC, KIRC, LIHC, PAAD, STAD, TGCT, THCA and UCEC. Importantly, in BRCA, HNSC, LIHC, PAAD, STAD, TGCT and UCEC, the 
methylation level of NSUN5 promoter was downregulated, while the expression of NSUN5 was upregulated. Notably, the methylation level of NSUN5 promoter had a trend of downregulation in BLCA, CESC, ESCA, KIRP, LUAD, LUSC, PRAD, SARC, SKCM and THYM, although it was not significant. It may be due to the small quantity of normal tissues. (Fig. 2)

To sum up, the methylation level of NSUN5 promoter was generally downregulated in cancers, and may contribute to the upregulation of NSUN5, especially for BRCA, HNSC, LIHC, PAAD, STAD, TGCT and UCEC.

\section{Higher expression of NSUN5 predicts poorer prognosis in multiple cancers}

We then used GEPIA2 to explore whether the expression of NSUN5 in cancers was related with the prognosis of cancer patients. Among the 33 types of cancers (ACC, BLCA, BRCA, CESC, CHOL, COAD, DLBC, ESCA, GBM, HNSC, KICH, KIRC, KIRP, LAML, LGG, LIHC, LUAD, LUSC, OV, PAAD, PCPG, PRAD, READ, SARC, SKCM, STAD, TGCT, THCA, THYM, UCEC, UCS and UVM (uveal melanoma)) with prognosis data in GEPIA2, higher expression of NSUN5 predicts lower OS (overall survival) for ACC $(P=0.0040)$, GBM $(P=0.0018)$, KIRC $(P=0.0079)$, LGG $(P=0.0060)$ and PRAD $(P=0.0330)$ patients, (Fig. 3)but predicts higher OS for SARC $(P=0.0420)$ and THYM $(P=0.0220)$ patients. (data not shown) The expression of NSUN5 was not associated with OS for the other 26 types of cancers. $(P>0.05)$ Furthermore, higher expression of NSUN5 predicts lower RFS (recurrence free survival) for $A C C(P=0.0140), L G G(P=0.0140), L I H C(P=$ 0.0055), LUSC $(P=0.0500)$ and PRAD $(P=0.0180)$ patients. (Fig. 4) The expression of NSUN5 was not associated with RFS for the other 28 types of cancers. $(P>0.05)$

Totally, higher expression of NSUN5 predicts poorer prognosis for ACC, LGG, PRAD (both for OS and RFS), GBM, KIRC (for OS), LIHC and LUSC (for RFS) patients.

\section{Higher expression of NSUN5 was positively correlated with translation in cancers}

To explore the function of NSUN5 in cancers, using LinkedOmics ${ }^{[10]}$ (http://linkedomics.org/login.php), GO (gene ontology) and KEGG (Kyoto Encyclopedia of Genes and Genomes) pathway analyses of NSUN5-correlated genes was performed in each cancer one by one. For example, in ACC, the co-expression analysis of NSUN5 (mRNA sequencing data) was shown in Supplementary Table 1 and Fig. 5A; the top 50 most significantly (according to FDR (false discovery rate)) NSUN5-positively-correlated and NSUN5-negatively-correlated genes were listed in Fig. 5B; the BPs (biological processes), CCs (cellular components), MFs (molecular functions) and KEGG pathways in which NSUN5-positively-correlated genes (FDR $\leq 0.05$ and normalized enrichment score $>0$ ) and NSUN5-negatively-correlated (FDR $\leq 0.05$ and normalized enrichment score $<0$ ) genes were significantly enriched were shown in Fig. 5C. The analyses process of the other 31 types of cancers were similar to that of ACC (data not shown).

The BPs, CCs, MFs and KEGG pathways in which NSUN5-positively correlated genes and NSUN5-negatively-correlated genes were significantly enriched in all the 32 types of human cancers were listed in Supplementary Tables 2-9.

To found the common BPs, CCs, MFs or KEGG pathways in human cancers, the number of cancer types with a same BP, CC, MF or KEGG pathway was calculated. The BPs, CCs, MFs or KEGG pathways were ranked by the times they appeared in cancers. The top 10 BPs, CCs, MFs and KEGG pathways in which NSUN5-positively-correlated genes were significantly enriched were listed in Tables 2-5, respectively. The top 10 BPs, CCs, MFs and KEGG pathways in which NSUN5-negatively-correlated genes were significantly enriched were listed in Supplementary Tables 10-13, respectively. 
Table 2

The top 10 BPs in which NSUN5-positively-correlated genes were significantly enriched in cancers.

\begin{tabular}{|c|c|c|c|c|c|c|c|c|c|}
\hline $\begin{array}{l}\text { BP } \\
\text { Cancers }\end{array}$ & $\begin{array}{l}\text { NADH } \\
\text { dehydrogenase } \\
\text { complex } \\
\text { assembly }\end{array}$ & $\begin{array}{l}\text { translational } \\
\text { elongation }\end{array}$ & $\begin{array}{l}\text { mitochondrial } \\
\text { respiratory } \\
\text { chain } \\
\text { complex } \\
\text { assembly }\end{array}$ & $\begin{array}{l}\text { rRNA } \\
\text { metabolic } \\
\text { process }\end{array}$ & $\begin{array}{l}\text { ncRNA } \\
\text { processing }\end{array}$ & $\begin{array}{l}\text { nucleoside } \\
\text { monophosphate } \\
\text { metabolic } \\
\text { process }\end{array}$ & $\begin{array}{l}\text { nucleoside } \\
\text { triphosphate } \\
\text { metabolic } \\
\text { process }\end{array}$ & $\begin{array}{l}\text { ribonucleoprotein } \\
\text { complex } \\
\text { biogenesis }\end{array}$ & $\begin{array}{l}\text { trans } \\
\text { initie }\end{array}$ \\
\hline ACC & $\sqrt{ }$ & $\sqrt{ }$ & $\sqrt{ }$ & $\sqrt{ }$ & $\sqrt{ }$ & $\sqrt{ }$ & $\sqrt{ }$ & $\sqrt{ }$ & $\sqrt{ }$ \\
\hline BLCA & $\sqrt{ }$ & $\sqrt{ }$ & $\sqrt{ }$ & $\sqrt{ }$ & $\sqrt{ }$ & $\sqrt{ }$ & $\sqrt{ }$ & $\sqrt{ }$ & $\sqrt{ }$ \\
\hline BRCA & $x$ & $x$ & $x$ & $x$ & $\sqrt{ }$ & $x$ & $x$ & $x$ & $x$ \\
\hline CESC & $\sqrt{ }$ & $\sqrt{ }$ & $\sqrt{ }$ & $\sqrt{ }$ & $\sqrt{ }$ & $\sqrt{ }$ & $\sqrt{ }$ & $\sqrt{ }$ & $\sqrt{ }$ \\
\hline $\mathrm{CHOL}$ & $\sqrt{ }$ & $\sqrt{ }$ & $\sqrt{ }$ & $\sqrt{ }$ & $\sqrt{ }$ & $\sqrt{ }$ & $\sqrt{ }$ & $\sqrt{ }$ & $\sqrt{ }$ \\
\hline COADREAD & $\sqrt{ }$ & $\sqrt{ }$ & $\sqrt{ }$ & $\sqrt{ }$ & $\sqrt{ }$ & $\sqrt{ }$ & $\sqrt{ }$ & $\sqrt{ }$ & $\sqrt{ }$ \\
\hline DLBC & $\sqrt{ }$ & $\sqrt{ }$ & $\sqrt{ }$ & $\sqrt{ }$ & $\sqrt{ }$ & $\sqrt{ }$ & $\sqrt{ }$ & $\sqrt{ }$ & $\sqrt{ }$ \\
\hline ESCA & $\sqrt{ }$ & $\sqrt{ }$ & $x$ & $x$ & $x$ & $\sqrt{ }$ & $\sqrt{ }$ & $x$ & $\sqrt{ }$ \\
\hline GBM & $\sqrt{ }$ & $\sqrt{ }$ & $\sqrt{ }$ & $x$ & $x$ & $\sqrt{ }$ & $\sqrt{ }$ & $x$ & $x$ \\
\hline HNSC & $x$ & $x$ & $x$ & $x$ & $x$ & $x$ & $x$ & $x$ & $x$ \\
\hline $\mathrm{KICH}$ & $\sqrt{ }$ & $\sqrt{ }$ & $\sqrt{ }$ & $\sqrt{ }$ & $\sqrt{ }$ & $x$ & $x$ & $\sqrt{ }$ & $\sqrt{ }$ \\
\hline KIRC & $\sqrt{ }$ & $\sqrt{ }$ & $\sqrt{ }$ & $\sqrt{ }$ & $\sqrt{ }$ & $\sqrt{ }$ & $\sqrt{ }$ & $\sqrt{ }$ & $\sqrt{ }$ \\
\hline KIRP & $\sqrt{ }$ & $\sqrt{ }$ & $\sqrt{ }$ & $\sqrt{ }$ & $\sqrt{ }$ & $x$ & $x$ & $\sqrt{ }$ & $x$ \\
\hline LAML & $\sqrt{ }$ & $\sqrt{ }$ & $\sqrt{ }$ & $\sqrt{ }$ & $\sqrt{ }$ & $\sqrt{ }$ & $\sqrt{ }$ & $\sqrt{ }$ & $\sqrt{ }$ \\
\hline LGG & $\sqrt{ }$ & $x$ & $\sqrt{ }$ & $x$ & $x$ & $x$ & $x$ & $x$ & $x$ \\
\hline LIHC & $\sqrt{ }$ & $\sqrt{ }$ & $\sqrt{ }$ & $\sqrt{ }$ & $\sqrt{ }$ & $\sqrt{ }$ & $\sqrt{ }$ & $\sqrt{ }$ & $\sqrt{ }$ \\
\hline LUAD & $\sqrt{ }$ & $\sqrt{ }$ & $\sqrt{ }$ & $\sqrt{ }$ & $\sqrt{ }$ & $\sqrt{ }$ & $\sqrt{ }$ & $\sqrt{ }$ & $x$ \\
\hline LUSC & $\sqrt{ }$ & $\sqrt{ }$ & $\sqrt{ }$ & $\sqrt{ }$ & $\sqrt{ }$ & $\sqrt{ }$ & $\sqrt{ }$ & $\sqrt{ }$ & $\sqrt{ }$ \\
\hline MESO & $\sqrt{ }$ & $\sqrt{ }$ & $\sqrt{ }$ & $\sqrt{ }$ & $\sqrt{ }$ & $\sqrt{ }$ & $\sqrt{ }$ & $\sqrt{ }$ & $\sqrt{ }$ \\
\hline OV & $\sqrt{ }$ & $\sqrt{ }$ & $\sqrt{ }$ & $\sqrt{ }$ & $\sqrt{ }$ & $\sqrt{ }$ & $\sqrt{ }$ & $\sqrt{ }$ & $x$ \\
\hline PAAD & $\sqrt{ }$ & $\sqrt{ }$ & $\sqrt{ }$ & $\sqrt{ }$ & $\sqrt{ }$ & $\sqrt{ }$ & $\sqrt{ }$ & $\sqrt{ }$ & $\sqrt{ }$ \\
\hline PCPG & $\sqrt{ }$ & $\sqrt{ }$ & $\sqrt{ }$ & $\sqrt{ }$ & $\sqrt{ }$ & $\sqrt{ }$ & $\sqrt{ }$ & $\sqrt{ }$ & $\sqrt{ }$ \\
\hline PRAD & $\sqrt{ }$ & $\sqrt{ }$ & $\sqrt{ }$ & $\sqrt{ }$ & $\sqrt{ }$ & $\sqrt{ }$ & $\sqrt{ }$ & $\sqrt{ }$ & $\sqrt{ }$ \\
\hline SARC & $\sqrt{ }$ & $\sqrt{ }$ & $\sqrt{ }$ & $\sqrt{ }$ & $\sqrt{ }$ & $\sqrt{ }$ & $\sqrt{ }$ & $\sqrt{ }$ & $\sqrt{ }$ \\
\hline SKCM & $\sqrt{ }$ & $\sqrt{ }$ & $\sqrt{ }$ & $\sqrt{ }$ & $\sqrt{ }$ & $\sqrt{ }$ & $\sqrt{ }$ & $\sqrt{ }$ & $\sqrt{ }$ \\
\hline STAD & $\sqrt{ }$ & $\sqrt{ }$ & $\sqrt{ }$ & $\sqrt{ }$ & $x$ & $x$ & $x$ & $\sqrt{ }$ & $\sqrt{ }$ \\
\hline TGCT & $\sqrt{ }$ & $\sqrt{ }$ & $x$ & $\sqrt{ }$ & $x$ & $\sqrt{ }$ & $\sqrt{ }$ & $x$ & $\sqrt{ }$ \\
\hline THCA & $\sqrt{ }$ & $\sqrt{ }$ & $\sqrt{ }$ & $\sqrt{ }$ & $\sqrt{ }$ & $\sqrt{ }$ & $\sqrt{ }$ & $\sqrt{ }$ & $\sqrt{ }$ \\
\hline THYM & $\sqrt{ }$ & $\sqrt{ }$ & $\sqrt{ }$ & $\sqrt{ }$ & $\sqrt{ }$ & $\sqrt{ }$ & $\sqrt{ }$ & $\sqrt{ }$ & $\sqrt{ }$ \\
\hline UCEC & $\sqrt{ }$ & $\sqrt{ }$ & $\sqrt{ }$ & $\sqrt{ }$ & $\sqrt{ }$ & $\sqrt{ }$ & $\sqrt{ }$ & $\sqrt{ }$ & $\sqrt{ }$ \\
\hline UCS & $\sqrt{ }$ & $\sqrt{ }$ & $\sqrt{ }$ & $\sqrt{ }$ & $\sqrt{ }$ & $\sqrt{ }$ & $\sqrt{ }$ & $\sqrt{ }$ & $\sqrt{ }$ \\
\hline UVM & $\sqrt{ }$ & $\sqrt{ }$ & $\sqrt{ }$ & $\sqrt{ }$ & $\sqrt{ }$ & $\sqrt{ }$ & $\sqrt{ }$ & $\sqrt{ }$ & $\sqrt{ }$ \\
\hline $\begin{array}{l}\text { Number of } \\
\text { cancer } \\
\text { types with } \\
\text { " }\end{array}$ & 30 & 29 & 28 & 27 & 26 & 26 & 26 & 26 & 25 \\
\hline
\end{tabular}

BP analyses of NSUN5-correlated genes in cancers using Linkedomics. The BP in which NSUN5-positively-correlated genes were significantly enriched (FDR $\leq$ normalized enrichment score $>0$ ) was denoted by " $\sqrt{ }$ ", otherwise, it was denoted by " $x$ ". The BPs were ranked by the number of cancer types with " $\sqrt{ }$ " and only shown. BP, biological process.

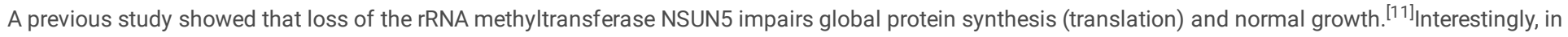
this study, we found that higher expression of NSUN5 in most cancers was positively correlated with translational elongation, rRNA metabolic process,

ribonucleoprotein complex biogenesis, translational initiation, (Table 2) ribosome, (Tables 3 and 5) rRNA binding and unfolded protein binding, (Table 4) which 
were all associated with protein synthesis. For example, in ACC, higher expression of NSUN5 was positively correlated with translational elongation, rRNA metabolic process, ribonucleoprotein complex biogenesis, translational initiation and ribosome. (Table 6) The corresponding gene (mRNA) symbols were listed in Table 6.

Table 3

The top $10 \mathrm{CCs}$ in which NSUN5-positively-correlated genes were significantly enriched in cancers.

\begin{tabular}{|c|c|c|c|c|c|c|c|c|c|}
\hline $\begin{array}{l}\text { CC } \\
\text { Cancers }\end{array}$ & $\begin{array}{l}\text { mitochondrial } \\
\text { inner } \\
\text { membrane }\end{array}$ & $\begin{array}{l}\text { mitochondrial } \\
\text { membrane } \\
\text { part }\end{array}$ & $\begin{array}{l}\text { mitochondrial } \\
\text { protein } \\
\text { complex }\end{array}$ & $\begin{array}{l}\text { NADH } \\
\text { dehydrogenase } \\
\text { complex }\end{array}$ & $\begin{array}{l}\text { organelle } \\
\text { envelope } \\
\text { lumen }\end{array}$ & $\begin{array}{l}\text { respiratory } \\
\text { chain }\end{array}$ & ribosome & $\begin{array}{l}\text { cytochrome } \\
\text { complex }\end{array}$ & $\begin{array}{l}\text { mitochondrial } \\
\text { matrix }\end{array}$ \\
\hline ACC & $\sqrt{ }$ & $\sqrt{ }$ & $\sqrt{ }$ & $\sqrt{ }$ & $\sqrt{ }$ & $\sqrt{ }$ & $\sqrt{ }$ & $\sqrt{ }$ & $x$ \\
\hline BLCA & $\sqrt{ }$ & $\sqrt{ }$ & $\sqrt{ }$ & $\sqrt{ }$ & $\sqrt{ }$ & $\sqrt{ }$ & $\sqrt{ }$ & $\sqrt{ }$ & $\sqrt{ }$ \\
\hline BRCA & $\sqrt{ }$ & $x$ & $x$ & $x$ & $x$ & $x$ & $x$ & $x$ & $x$ \\
\hline CESC & $\sqrt{ }$ & $\sqrt{ }$ & $\sqrt{ }$ & $\sqrt{ }$ & $\sqrt{ }$ & $\sqrt{ }$ & $\sqrt{ }$ & $\sqrt{ }$ & $\sqrt{ }$ \\
\hline $\mathrm{CHOL}$ & $\sqrt{ }$ & $\sqrt{ }$ & $\sqrt{ }$ & $\sqrt{ }$ & $\sqrt{ }$ & $\sqrt{ }$ & $\sqrt{ }$ & $\sqrt{ }$ & $\sqrt{ }$ \\
\hline COADREAD & $\sqrt{ }$ & $\sqrt{ }$ & $\sqrt{ }$ & $\sqrt{ }$ & $\sqrt{ }$ & $\sqrt{ }$ & $\sqrt{ }$ & $\sqrt{ }$ & $\sqrt{ }$ \\
\hline DLBC & $\sqrt{ }$ & $\sqrt{ }$ & $\sqrt{ }$ & $\sqrt{ }$ & $\sqrt{ }$ & $\sqrt{ }$ & $\sqrt{ }$ & $\sqrt{ }$ & $\sqrt{ }$ \\
\hline ESCA & $\sqrt{ }$ & $\sqrt{ }$ & $\sqrt{ }$ & $\sqrt{ }$ & $\sqrt{ }$ & $\sqrt{ }$ & $\sqrt{ }$ & $\sqrt{ }$ & $\sqrt{ }$ \\
\hline GBM & $\sqrt{ }$ & $\sqrt{ }$ & $\sqrt{ }$ & $\sqrt{ }$ & $\sqrt{ }$ & $\sqrt{ }$ & $\sqrt{ }$ & $x$ & $\sqrt{ }$ \\
\hline HNSC & $\sqrt{ }$ & $\sqrt{ }$ & $\sqrt{ }$ & $\sqrt{ }$ & $\sqrt{ }$ & $\sqrt{ }$ & $\sqrt{ }$ & $\sqrt{ }$ & $\sqrt{ }$ \\
\hline $\mathrm{KICH}$ & $\sqrt{ }$ & $\sqrt{ }$ & $\sqrt{ }$ & $\sqrt{ }$ & $\sqrt{ }$ & $\sqrt{ }$ & $\sqrt{ }$ & $\sqrt{ }$ & $\sqrt{ }$ \\
\hline KIRC & $\sqrt{ }$ & $\sqrt{ }$ & $\sqrt{ }$ & $\sqrt{ }$ & $\sqrt{ }$ & $\sqrt{ }$ & $\sqrt{ }$ & $\sqrt{ }$ & $\sqrt{ }$ \\
\hline KIRP & $\sqrt{ }$ & $\sqrt{ }$ & $\sqrt{ }$ & $\sqrt{ }$ & $\sqrt{ }$ & $\sqrt{ }$ & $\sqrt{ }$ & $\sqrt{ }$ & $\sqrt{ }$ \\
\hline LAML & $\sqrt{ }$ & $\sqrt{ }$ & $\sqrt{ }$ & $\sqrt{ }$ & $\sqrt{ }$ & $\sqrt{ }$ & $\sqrt{ }$ & $\sqrt{ }$ & $\sqrt{ }$ \\
\hline LGG & $\sqrt{ }$ & $\sqrt{ }$ & $\sqrt{ }$ & $\sqrt{ }$ & $\sqrt{ }$ & $\sqrt{ }$ & $x$ & $\sqrt{ }$ & $x$ \\
\hline LIHC & $\sqrt{ }$ & $\sqrt{ }$ & $\sqrt{ }$ & $\sqrt{ }$ & $\sqrt{ }$ & $\sqrt{ }$ & $\sqrt{ }$ & $\sqrt{ }$ & $\sqrt{ }$ \\
\hline LUAD & $\sqrt{ }$ & $\sqrt{ }$ & $\sqrt{ }$ & $\sqrt{ }$ & $\sqrt{ }$ & $\sqrt{ }$ & $\sqrt{ }$ & $\sqrt{ }$ & $\sqrt{ }$ \\
\hline LUSC & $\sqrt{ }$ & $\sqrt{ }$ & $\sqrt{ }$ & $\sqrt{ }$ & $\sqrt{ }$ & $\sqrt{ }$ & $\sqrt{ }$ & $\sqrt{ }$ & $\sqrt{ }$ \\
\hline MESO & $\sqrt{ }$ & $\sqrt{ }$ & $\sqrt{ }$ & $\sqrt{ }$ & $\sqrt{ }$ & $\sqrt{ }$ & $\sqrt{ }$ & $\sqrt{ }$ & $\sqrt{ }$ \\
\hline ov & $\sqrt{ }$ & $\sqrt{ }$ & $\sqrt{ }$ & $\sqrt{ }$ & $\sqrt{ }$ & $\sqrt{ }$ & $\sqrt{ }$ & $x$ & $\sqrt{ }$ \\
\hline PAAD & $\sqrt{ }$ & $\sqrt{ }$ & $\sqrt{ }$ & $\sqrt{ }$ & $\sqrt{ }$ & $\sqrt{ }$ & $\sqrt{ }$ & $\sqrt{ }$ & $\sqrt{ }$ \\
\hline PCPG & $\sqrt{ }$ & $\sqrt{ }$ & $\sqrt{ }$ & $\sqrt{ }$ & $\sqrt{ }$ & $\sqrt{ }$ & $\sqrt{ }$ & $\sqrt{ }$ & $\sqrt{ }$ \\
\hline PRAD & $\sqrt{ }$ & $\sqrt{ }$ & $\sqrt{ }$ & $\sqrt{ }$ & $\sqrt{ }$ & $\sqrt{ }$ & $\sqrt{ }$ & $\sqrt{ }$ & $\sqrt{ }$ \\
\hline SARC & $\sqrt{ }$ & $\sqrt{ }$ & $\sqrt{ }$ & $\sqrt{ }$ & $\sqrt{ }$ & $\sqrt{ }$ & $\sqrt{ }$ & $\sqrt{ }$ & $\sqrt{ }$ \\
\hline SKCM & $\sqrt{ }$ & $\sqrt{ }$ & $\sqrt{ }$ & $\sqrt{ }$ & $\sqrt{ }$ & $\sqrt{ }$ & $\sqrt{ }$ & $\sqrt{ }$ & $\sqrt{ }$ \\
\hline STAD & $\sqrt{ }$ & $\sqrt{ }$ & $\sqrt{ }$ & $\sqrt{ }$ & $\sqrt{ }$ & $\sqrt{ }$ & $\sqrt{ }$ & $\sqrt{ }$ & $\sqrt{ }$ \\
\hline TGCT & $\sqrt{ }$ & $\sqrt{ }$ & $\sqrt{ }$ & $\sqrt{ }$ & $\sqrt{ }$ & $\sqrt{ }$ & $\sqrt{ }$ & $\sqrt{ }$ & $\sqrt{ }$ \\
\hline THCA & $\sqrt{ }$ & $\sqrt{ }$ & $\sqrt{ }$ & $\sqrt{ }$ & $\sqrt{ }$ & $\sqrt{ }$ & $\sqrt{ }$ & $\sqrt{ }$ & $\sqrt{ }$ \\
\hline THYM & $\sqrt{ }$ & $\sqrt{ }$ & $\sqrt{ }$ & $\sqrt{ }$ & $\sqrt{ }$ & $\sqrt{ }$ & $\sqrt{ }$ & $\sqrt{ }$ & $\sqrt{ }$ \\
\hline UCEC & $\sqrt{ }$ & $\sqrt{ }$ & $\sqrt{ }$ & $\sqrt{ }$ & $\sqrt{ }$ & $\sqrt{ }$ & $\sqrt{ }$ & $\sqrt{ }$ & $\sqrt{ }$ \\
\hline UCS & $\sqrt{ }$ & $\sqrt{ }$ & $\sqrt{ }$ & $\sqrt{ }$ & $\sqrt{ }$ & $\sqrt{ }$ & $\sqrt{ }$ & $\sqrt{ }$ & $\sqrt{ }$ \\
\hline UVM & $\sqrt{ }$ & $\sqrt{ }$ & $\sqrt{ }$ & $\sqrt{ }$ & $\sqrt{ }$ & $\sqrt{ }$ & $\sqrt{ }$ & $\sqrt{ }$ & $\sqrt{ }$ \\
\hline $\begin{array}{l}\text { Number of } \\
\text { cancer } \\
\text { types with } \\
\text { " } " \text { " }\end{array}$ & 32 & 31 & 31 & 31 & 31 & 31 & 30 & 29 & 29 \\
\hline
\end{tabular}

CC analyses of NSUN5-correlated genes in cancers using Linkedomics. The CC in which NSUN5-positively-correlated genes were significantly enriched (FDR $\leq$ normalized enrichment score $>0$ ) was denoted by " $\sqrt{ }$ ", otherwise, it was denoted by " $x$ ". The CCs were ranked by the number of cancer types with " $\sqrt{ }$ " and onls were shown. CC, cellular component. 
Table 4

The top 10 MFs in which NSUN5-positively-correlated genes were significantly enriched in cancers.

\begin{tabular}{|c|c|c|c|c|c|c|c|c|c|c|c|}
\hline $\begin{array}{l}\text { MF } \\
\text { Cancers }\end{array}$ & $\begin{array}{l}\text { oxidoreductase } \\
\text { activity, acting } \\
\text { on a heme } \\
\text { group of } \\
\text { donors }\end{array}$ & $\begin{array}{l}\text { structural } \\
\text { constituent } \\
\text { of } \\
\text { ribosome }\end{array}$ & $\begin{array}{l}\text { electron } \\
\text { transfer } \\
\text { activity }\end{array}$ & $\begin{array}{l}\text { oxidoreductase } \\
\text { activity, acting } \\
\text { on } N A D(P) H\end{array}$ & $\begin{array}{l}\text { heme- } \\
\text { copper } \\
\text { terminal } \\
\text { oxidase } \\
\text { activity }\end{array}$ & $\begin{array}{l}\text { rRNA } \\
\text { binding }\end{array}$ & $\begin{array}{l}\text { threonine- } \\
\text { type } \\
\text { peptidase } \\
\text { activity }\end{array}$ & $\begin{array}{l}\text { catalytic } \\
\text { activity, } \\
\text { acting } \\
\text { on DNA }\end{array}$ & $\begin{array}{l}\text { metal } \\
\text { cluster } \\
\text { binding }\end{array}$ & $\begin{array}{l}\text { snRNA } \\
\text { binding }\end{array}$ & $\begin{array}{l}\text { unfolde } \\
\text { protein } \\
\text { binding }\end{array}$ \\
\hline ACC & $x$ & $x$ & $x$ & $x$ & $x$ & $x$ & $x$ & $x$ & $x$ & $x$ & $x$ \\
\hline BLCA & $\sqrt{ }$ & $\sqrt{ }$ & $\sqrt{ }$ & $\sqrt{ }$ & $\sqrt{ }$ & $\sqrt{ }$ & $\sqrt{ }$ & $\sqrt{ }$ & $\sqrt{ }$ & $\sqrt{ }$ & $\sqrt{ }$ \\
\hline BRCA & $\sqrt{ }$ & $x$ & $x$ & $x$ & $x$ & $x$ & $x$ & $\sqrt{ }$ & $x$ & $x$ & $x$ \\
\hline CESC & $\sqrt{ }$ & $\sqrt{ }$ & $\sqrt{ }$ & $\sqrt{ }$ & $\sqrt{ }$ & $\sqrt{ }$ & $\sqrt{ }$ & $\sqrt{ }$ & $\sqrt{ }$ & $\sqrt{ }$ & $\sqrt{ }$ \\
\hline $\mathrm{CHOL}$ & $\sqrt{ }$ & $\sqrt{ }$ & $\sqrt{ }$ & $\sqrt{ }$ & $\sqrt{ }$ & $\sqrt{ }$ & $\sqrt{ }$ & $\sqrt{ }$ & $\sqrt{ }$ & $\sqrt{ }$ & $\sqrt{ }$ \\
\hline COADREAD & $\sqrt{ }$ & $\sqrt{ }$ & $\sqrt{ }$ & $\sqrt{ }$ & $\sqrt{ }$ & $\sqrt{ }$ & $\sqrt{ }$ & $\sqrt{ }$ & $\sqrt{ }$ & $\sqrt{ }$ & $\sqrt{ }$ \\
\hline DLBC & $\sqrt{ }$ & $\sqrt{ }$ & $\sqrt{ }$ & $\sqrt{ }$ & $\sqrt{ }$ & $\sqrt{ }$ & $\sqrt{ }$ & $\sqrt{ }$ & $\sqrt{ }$ & $\sqrt{ }$ & $\sqrt{ }$ \\
\hline ESCA & $\sqrt{ }$ & $\sqrt{ }$ & $\sqrt{ }$ & $\sqrt{ }$ & $\sqrt{ }$ & $\sqrt{ }$ & $\sqrt{ }$ & $\sqrt{ }$ & $\sqrt{ }$ & $x$ & $\sqrt{ }$ \\
\hline GBM & $\sqrt{ }$ & $\sqrt{ }$ & $\sqrt{ }$ & $\sqrt{ }$ & $x$ & $x$ & $\sqrt{ }$ & $x$ & $x$ & $x$ & $\sqrt{ }$ \\
\hline HNSC & $\sqrt{ }$ & $\sqrt{ }$ & $\sqrt{ }$ & $\sqrt{ }$ & $\sqrt{ }$ & $\sqrt{ }$ & $\sqrt{ }$ & $\sqrt{ }$ & $\sqrt{ }$ & $\sqrt{ }$ & $\sqrt{ }$ \\
\hline $\mathrm{KICH}$ & $x$ & $\sqrt{ }$ & $x$ & $x$ & $x$ & $\sqrt{ }$ & $\sqrt{ }$ & $x$ & $x$ & $x$ & $x$ \\
\hline KIRC & $\sqrt{ }$ & $\sqrt{ }$ & $\sqrt{ }$ & $\sqrt{ }$ & $\sqrt{ }$ & $\sqrt{ }$ & $\sqrt{ }$ & $\sqrt{ }$ & $\sqrt{ }$ & $\sqrt{ }$ & $\sqrt{ }$ \\
\hline KIRP & $\sqrt{ }$ & $\sqrt{ }$ & $\sqrt{ }$ & $\sqrt{ }$ & $\sqrt{ }$ & $\sqrt{ }$ & $\sqrt{ }$ & $x$ & $x$ & $\sqrt{ }$ & $x$ \\
\hline LAML & $\sqrt{ }$ & $\sqrt{ }$ & $\sqrt{ }$ & $\sqrt{ }$ & $\sqrt{ }$ & $\sqrt{ }$ & $\sqrt{ }$ & $\sqrt{ }$ & $\sqrt{ }$ & $\sqrt{ }$ & $\sqrt{ }$ \\
\hline LGG & $\sqrt{ }$ & $x$ & $\sqrt{ }$ & $\sqrt{ }$ & $\sqrt{ }$ & $x$ & $\sqrt{ }$ & $x$ & $x$ & $x$ & $\sqrt{ }$ \\
\hline LIHC & $\sqrt{ }$ & $\sqrt{ }$ & $\sqrt{ }$ & $\sqrt{ }$ & $\sqrt{ }$ & $\sqrt{ }$ & $\sqrt{ }$ & $\sqrt{ }$ & $x$ & $\sqrt{ }$ & $\sqrt{ }$ \\
\hline LUAD & $\sqrt{ }$ & $\sqrt{ }$ & $\sqrt{ }$ & $\sqrt{ }$ & $\sqrt{ }$ & $\sqrt{ }$ & $\sqrt{ }$ & $\sqrt{ }$ & $\sqrt{ }$ & $\sqrt{ }$ & $\sqrt{ }$ \\
\hline LUSC & $\sqrt{ }$ & $\sqrt{ }$ & $\sqrt{ }$ & $\sqrt{ }$ & $\sqrt{ }$ & $\sqrt{ }$ & $\sqrt{ }$ & $\sqrt{ }$ & $\sqrt{ }$ & $\sqrt{ }$ & $\sqrt{ }$ \\
\hline MESO & $\sqrt{ }$ & $\sqrt{ }$ & $\sqrt{ }$ & $\sqrt{ }$ & $\sqrt{ }$ & $\sqrt{ }$ & $\sqrt{ }$ & $\sqrt{ }$ & $\sqrt{ }$ & $\sqrt{ }$ & $\sqrt{ }$ \\
\hline OV & $\sqrt{ }$ & $\sqrt{ }$ & $\sqrt{ }$ & $\sqrt{ }$ & $x$ & $x$ & $x$ & $\sqrt{ }$ & $\sqrt{ }$ & $x$ & $\sqrt{ }$ \\
\hline PAAD & $\sqrt{ }$ & $\sqrt{ }$ & $\sqrt{ }$ & $\sqrt{ }$ & $\sqrt{ }$ & $\sqrt{ }$ & $\sqrt{ }$ & $\sqrt{ }$ & $\sqrt{ }$ & $\sqrt{ }$ & $\sqrt{ }$ \\
\hline PCPG & $\sqrt{ }$ & $\sqrt{ }$ & $\sqrt{ }$ & $\sqrt{ }$ & $\sqrt{ }$ & $\sqrt{ }$ & $\sqrt{ }$ & $\sqrt{ }$ & $\sqrt{ }$ & $\sqrt{ }$ & $x$ \\
\hline PRAD & $\sqrt{ }$ & $\sqrt{ }$ & $\sqrt{ }$ & $\sqrt{ }$ & $\sqrt{ }$ & $\sqrt{ }$ & $\sqrt{ }$ & $\sqrt{ }$ & $\sqrt{ }$ & $\sqrt{ }$ & $\sqrt{ }$ \\
\hline SARC & $\sqrt{ }$ & $\sqrt{ }$ & $\sqrt{ }$ & $\sqrt{ }$ & $\sqrt{ }$ & $\sqrt{ }$ & $\sqrt{ }$ & $\sqrt{ }$ & $\sqrt{ }$ & $\sqrt{ }$ & $\sqrt{ }$ \\
\hline SKCM & $\sqrt{ }$ & $\sqrt{ }$ & $\sqrt{ }$ & $\sqrt{ }$ & $\sqrt{ }$ & $\sqrt{ }$ & $x$ & $x$ & $\sqrt{ }$ & $\sqrt{ }$ & $x$ \\
\hline STAD & $\sqrt{ }$ & $\sqrt{ }$ & $\sqrt{ }$ & $x$ & $\sqrt{ }$ & $\sqrt{ }$ & $\sqrt{ }$ & $\sqrt{ }$ & $\sqrt{ }$ & $\sqrt{ }$ & $\sqrt{ }$ \\
\hline TGCT & $\sqrt{ }$ & $\sqrt{ }$ & $\sqrt{ }$ & $\sqrt{ }$ & $\sqrt{ }$ & $\sqrt{ }$ & $\sqrt{ }$ & $\sqrt{ }$ & $\sqrt{ }$ & $\sqrt{ }$ & $\sqrt{ }$ \\
\hline THCA & $\sqrt{ }$ & $\sqrt{ }$ & $\sqrt{ }$ & $\sqrt{ }$ & $\sqrt{ }$ & $\sqrt{ }$ & $\sqrt{ }$ & $x$ & $\sqrt{ }$ & $x$ & $x$ \\
\hline THYM & $\sqrt{ }$ & $\sqrt{ }$ & $\sqrt{ }$ & $\sqrt{ }$ & $\sqrt{ }$ & $\sqrt{ }$ & $\sqrt{ }$ & $\sqrt{ }$ & $\sqrt{ }$ & $\sqrt{ }$ & $\sqrt{ }$ \\
\hline UCEC & $\sqrt{ }$ & $\sqrt{ }$ & $\sqrt{ }$ & $\sqrt{ }$ & $\sqrt{ }$ & $\sqrt{ }$ & $\sqrt{ }$ & $\sqrt{ }$ & $\sqrt{ }$ & $\sqrt{ }$ & $\sqrt{ }$ \\
\hline UCS & $\sqrt{ }$ & $\sqrt{ }$ & $\sqrt{ }$ & $\sqrt{ }$ & $\sqrt{ }$ & $\sqrt{ }$ & $\sqrt{ }$ & $\sqrt{ }$ & $\sqrt{ }$ & $\sqrt{ }$ & $\sqrt{ }$ \\
\hline UVM & $\sqrt{ }$ & $\sqrt{ }$ & $x$ & $\sqrt{ }$ & $\sqrt{ }$ & $\sqrt{ }$ & $x$ & $x$ & $x$ & $\sqrt{ }$ & $x$ \\
\hline $\begin{array}{l}\text { Number of } \\
\text { cancer } \\
\text { types with } \\
\text { " } \sqrt{ } \text { " }\end{array}$ & 30 & 29 & 28 & 28 & 27 & 27 & 27 & 24 & 24 & 24 & 24 \\
\hline
\end{tabular}

MF analyses of NSUN5-correlated genes in cancers using Linkedomics. The MF in which NSUN5-positively-correlated genes were significantly enriched (FDR $\leq 0.05$ and normalized enrichment score $>0$ ) was denoted by " $\sqrt{ }$ ", otherwise, it was denoted by " $x$ ". The MFs were ranked by the number of cancer types with " $\checkmark$ " and only the top 10 MFs were shown. MF, molecular function.' 
Table 5

The top 10 pathways in which NSUN5-positively-correlated genes were significantly enriched in cancers.

\begin{tabular}{|c|c|c|c|c|c|c|c|c|c|c|}
\hline $\begin{array}{l}\text { Pathway } \\
\text { Cancers }\end{array}$ & $\begin{array}{l}\text { Oxidative } \\
\text { phosphorylation }\end{array}$ & $\begin{array}{l}\text { Parkinson } \\
\text { disease }\end{array}$ & $\begin{array}{l}\text { Huntington } \\
\text { disease }\end{array}$ & $\begin{array}{l}\text { Non- } \\
\text { alcoholic } \\
\text { fatty } \\
\text { liver } \\
\text { disease } \\
\text { (NAFLD) }\end{array}$ & Ribosome & $\begin{array}{l}\text { Pyrimidine } \\
\text { metabolism }\end{array}$ & Spliceosome & $\begin{array}{l}\text { Alzheimer } \\
\text { disease }\end{array}$ & $\begin{array}{l}\text { Base } \\
\text { excision } \\
\text { repair }\end{array}$ & Proteasom \\
\hline ACC & $\sqrt{ }$ & $\sqrt{ }$ & $\sqrt{ }$ & $\sqrt{ }$ & $\sqrt{ }$ & $\sqrt{ }$ & $\sqrt{ }$ & $\sqrt{ }$ & $\times$ & $x$ \\
\hline BLCA & $\sqrt{ }$ & $\sqrt{ }$ & $\sqrt{ }$ & $\sqrt{ }$ & $\sqrt{ }$ & $\sqrt{ }$ & $\sqrt{ }$ & $\sqrt{ }$ & $\sqrt{ }$ & $\sqrt{ }$ \\
\hline BRCA & $x$ & $x$ & $x$ & $x$ & $x$ & $x$ & $x$ & $x$ & $x$ & $x$ \\
\hline CESC & $\sqrt{ }$ & $\sqrt{ }$ & $\sqrt{ }$ & $\sqrt{ }$ & $\sqrt{ }$ & $\sqrt{ }$ & $\sqrt{ }$ & $\sqrt{ }$ & $\sqrt{ }$ & $\sqrt{ }$ \\
\hline $\mathrm{CHOL}$ & $\sqrt{ }$ & $\sqrt{ }$ & $\sqrt{ }$ & $\sqrt{ }$ & $\sqrt{ }$ & $\sqrt{ }$ & $\sqrt{ }$ & $\sqrt{ }$ & $\sqrt{ }$ & $\sqrt{ }$ \\
\hline COADREAD & $\sqrt{ }$ & $\sqrt{ }$ & $\sqrt{ }$ & $\sqrt{ }$ & $\sqrt{ }$ & $\sqrt{ }$ & $\sqrt{ }$ & $\sqrt{ }$ & $\sqrt{ }$ & $\sqrt{ }$ \\
\hline DLBC & $\sqrt{ }$ & $\sqrt{ }$ & $\sqrt{ }$ & $\sqrt{ }$ & $\sqrt{ }$ & $\sqrt{ }$ & $\sqrt{ }$ & $\sqrt{ }$ & $\sqrt{ }$ & $\sqrt{ }$ \\
\hline ESCA & $\sqrt{ }$ & $\sqrt{ }$ & $\sqrt{ }$ & $\sqrt{ }$ & $\sqrt{ }$ & $\sqrt{ }$ & $\sqrt{ }$ & $\sqrt{ }$ & $\sqrt{ }$ & $\sqrt{ }$ \\
\hline GBM & $\sqrt{ }$ & $\sqrt{ }$ & $\sqrt{ }$ & $x$ & $x$ & $\sqrt{ }$ & $x$ & $x$ & $\sqrt{ }$ & $\sqrt{ }$ \\
\hline HNSC & $\sqrt{ }$ & $\sqrt{ }$ & $\sqrt{ }$ & $\sqrt{ }$ & $\sqrt{ }$ & $\sqrt{ }$ & $\sqrt{ }$ & $\sqrt{ }$ & $\sqrt{ }$ & $\sqrt{ }$ \\
\hline $\mathrm{KICH}$ & $\sqrt{ }$ & $\sqrt{ }$ & $\sqrt{ }$ & $\sqrt{ }$ & $\sqrt{ }$ & $\sqrt{ }$ & $\sqrt{ }$ & $\sqrt{ }$ & $x$ & $x$ \\
\hline KIRC & $\sqrt{ }$ & $\sqrt{ }$ & $\sqrt{ }$ & $\sqrt{ }$ & $\sqrt{ }$ & $\sqrt{ }$ & $\sqrt{ }$ & $\sqrt{ }$ & $\sqrt{ }$ & $\sqrt{ }$ \\
\hline KIRP & $\sqrt{ }$ & $\sqrt{ }$ & $\sqrt{ }$ & $\sqrt{ }$ & $\sqrt{ }$ & $x$ & $\sqrt{ }$ & $x$ & $\sqrt{ }$ & $\sqrt{ }$ \\
\hline LAML & $\sqrt{ }$ & $\sqrt{ }$ & $\sqrt{ }$ & $\sqrt{ }$ & $\sqrt{ }$ & $\sqrt{ }$ & $x$ & $\sqrt{ }$ & $\sqrt{ }$ & $x$ \\
\hline LGG & $\sqrt{ }$ & $\sqrt{ }$ & $\times$ & $\sqrt{ }$ & $x$ & $x$ & $x$ & $\sqrt{ }$ & $\times$ & $\sqrt{ }$ \\
\hline LIHC & $\sqrt{ }$ & $\sqrt{ }$ & $\sqrt{ }$ & $\sqrt{ }$ & $\sqrt{ }$ & $\sqrt{ }$ & $\sqrt{ }$ & $\sqrt{ }$ & $\sqrt{ }$ & $\sqrt{ }$ \\
\hline LUAD & $\sqrt{ }$ & $\sqrt{ }$ & $\sqrt{ }$ & $\sqrt{ }$ & $\sqrt{ }$ & $\sqrt{ }$ & $\sqrt{ }$ & $\sqrt{ }$ & $\sqrt{ }$ & $\sqrt{ }$ \\
\hline LUSC & $\sqrt{ }$ & $\sqrt{ }$ & $\sqrt{ }$ & $\sqrt{ }$ & $\sqrt{ }$ & $\sqrt{ }$ & $\sqrt{ }$ & $x$ & $\sqrt{ }$ & $\sqrt{ }$ \\
\hline MESO & $\sqrt{ }$ & $\sqrt{ }$ & $\sqrt{ }$ & $\sqrt{ }$ & $\sqrt{ }$ & $\sqrt{ }$ & $\sqrt{ }$ & $\sqrt{ }$ & $\sqrt{ }$ & $\sqrt{ }$ \\
\hline OV & $\sqrt{ }$ & $\sqrt{ }$ & $\sqrt{ }$ & $x$ & $\sqrt{ }$ & $\sqrt{ }$ & $\sqrt{ }$ & $\sqrt{ }$ & $x$ & $\sqrt{ }$ \\
\hline PAAD & $\sqrt{ }$ & $\sqrt{ }$ & $\sqrt{ }$ & $\sqrt{ }$ & $\sqrt{ }$ & $\sqrt{ }$ & $\sqrt{ }$ & $\sqrt{ }$ & $\sqrt{ }$ & $\sqrt{ }$ \\
\hline PCPG & $\sqrt{ }$ & $\sqrt{ }$ & $\sqrt{ }$ & $\sqrt{ }$ & $\sqrt{ }$ & $\sqrt{ }$ & $\sqrt{ }$ & $\sqrt{ }$ & $\sqrt{ }$ & $\sqrt{ }$ \\
\hline PRAD & $\sqrt{ }$ & $\sqrt{ }$ & $\sqrt{ }$ & $\sqrt{ }$ & $\sqrt{ }$ & $\sqrt{ }$ & $\sqrt{ }$ & $\sqrt{ }$ & $\sqrt{ }$ & $\sqrt{ }$ \\
\hline SARC & $\sqrt{ }$ & $\sqrt{ }$ & $\sqrt{ }$ & $\sqrt{ }$ & $\sqrt{ }$ & $\sqrt{ }$ & $\sqrt{ }$ & $\sqrt{ }$ & $\sqrt{ }$ & $\sqrt{ }$ \\
\hline SKCM & $\sqrt{ }$ & $\sqrt{ }$ & $\sqrt{ }$ & $\sqrt{ }$ & $\sqrt{ }$ & $\sqrt{ }$ & $\sqrt{ }$ & $\sqrt{ }$ & $\sqrt{ }$ & $x$ \\
\hline STAD & $\sqrt{ }$ & $\sqrt{ }$ & $\sqrt{ }$ & $\sqrt{ }$ & $\sqrt{ }$ & $\sqrt{ }$ & $\sqrt{ }$ & $x$ & $\sqrt{ }$ & $\sqrt{ }$ \\
\hline TGCT & $\sqrt{ }$ & $\sqrt{ }$ & $\sqrt{ }$ & $\sqrt{ }$ & $\sqrt{ }$ & $\sqrt{ }$ & $\sqrt{ }$ & $\sqrt{ }$ & $x$ & $\sqrt{ }$ \\
\hline THCA & $\sqrt{ }$ & $\sqrt{ }$ & $\sqrt{ }$ & $\sqrt{ }$ & $\sqrt{ }$ & $\sqrt{ }$ & $\sqrt{ }$ & $\sqrt{ }$ & $\sqrt{ }$ & $\sqrt{ }$ \\
\hline THYM & $\sqrt{ }$ & $\sqrt{ }$ & $\sqrt{ }$ & $\sqrt{ }$ & $\sqrt{ }$ & $\sqrt{ }$ & $\sqrt{ }$ & $\sqrt{ }$ & $\sqrt{ }$ & $\sqrt{ }$ \\
\hline UCEC & $\sqrt{ }$ & $\sqrt{ }$ & $\sqrt{ }$ & $\sqrt{ }$ & $\sqrt{ }$ & $\sqrt{ }$ & $\sqrt{ }$ & $\sqrt{ }$ & $\sqrt{ }$ & $\sqrt{ }$ \\
\hline UCS & $\sqrt{ }$ & $\sqrt{ }$ & $\sqrt{ }$ & $\sqrt{ }$ & $\sqrt{ }$ & $\sqrt{ }$ & $\sqrt{ }$ & $\sqrt{ }$ & $\sqrt{ }$ & $\sqrt{ }$ \\
\hline UVM & $\sqrt{ }$ & $\sqrt{ }$ & $\sqrt{ }$ & $\sqrt{ }$ & $\sqrt{ }$ & $x$ & $\sqrt{ }$ & $\sqrt{ }$ & $\sqrt{ }$ & $x$ \\
\hline $\begin{array}{l}\text { Number of } \\
\text { cancer } \\
\text { types with } \\
\text { " } \sqrt{ } \text { " }\end{array}$ & 31 & 31 & 30 & 29 & 29 & 28 & 28 & 27 & 26 & 26 \\
\hline
\end{tabular}

KEGG pathway analyses of NSUN5-correlated genes in cancers using Linkedomics. The pathway in which NSUN5-positively-correlated genes were significantly enriched (FDR $\leq 0.05$ and normalized enrichment score $>0$ ) was denoted by " $\sqrt{ }$ ", otherwise, it was denoted by " $x$ ". The pathways were ranked by the number of cancer types with " $\sqrt{ }$ " and only the top 10 pathways were shown. KEGG, Kyoto Encyclopedia of Genes and Genomes. 
Table 6

The translation-associated BPs, CCs, MFs and KEGG pathways in which NSUN5-positively correlated genes were significantly enriched in ACC

\begin{tabular}{|c|c|c|c|c|}
\hline Gene set & & NES & FDR & Gene (mRNA) symbols \\
\hline \multirow[t]{5}{*}{ BP } & \multirow{5}{*}{$\begin{array}{l}\text { translational } \\
\text { elongation }\end{array}$} & \multirow[t]{5}{*}{1.708} & \multirow[t]{5}{*}{0.026} & AURKAIP1;EEF1A2;EEF1D;EEF2;EEF2K;EEFSEC;EIF5A;EIF5AL1;GADD45GIP1; \\
\hline & & & & MRPL11;MRPL14;MRPL20;MRPL21;MRPL22;MRPL27;MRPL3;MRPL32;MRPL34; \\
\hline & & & & MRPL36;MRPL4;MRPL41;MRPL42;MRPL47;MRPL51;MRPL52;MRPL54;MRPS11; \\
\hline & & & & MRPS12;MRPS15;MRPS17;MRPS2;MRPS21;MRPS24;MRPS26;MRPS33;MRPS34; \\
\hline & & & & MRPS36;PSTK;RPLP1;RPLP2;TUFM;ZNF598 \\
\hline \multirow[t]{7}{*}{ BP } & \multirow{7}{*}{$\begin{array}{l}\text { rRNA metabolic } \\
\text { process }\end{array}$} & \multirow[t]{7}{*}{1.962} & \multirow[t]{7}{*}{0.003} & BRF1;BYSL;CD3EAP;DDX27;DDX49;DDX56;DEDD2;DHX37;DKC1;EBNA1BP2; \\
\hline & & & & ERI3;ERN2;EXOSC1;EXOSC3;EXOSC5;FBL;FRG1;GAR1;GTF3A;GTF3C1;H2AFY; \\
\hline & & & & ISG20;KRI1;KRR1;LAS1L;LYAR;MARS;MRPS11;MRTO4;NGDN;NHP2;NOB1;NOC4L; \\
\hline & & & & NOL12;NOL6;NOL8;NOP10;NOP2;NOP56;NSA2;NSUN5;NVL;PA2G4;PIH1D1;POP4; \\
\hline & & & & RIOK2;RPF2;RPL10A;RPL11;RPL26;RPL35;RPL35A;RPL5;RPL7A;RPS14;RPS15;RPS16; \\
\hline & & & & RPS17;RPS19;RPS2;RPS21;RPS27;RPS28;RPS6;RPS7;RPS8;RPS9;RRP1;RRP9;RSL1D1; \\
\hline & & & & SART1;SBDS;SMARCA4;SUV39H1;TBL3;UTP14A;UTP15;UTP20;WDR36;WDR46;ZNHIT3 \\
\hline \multirow[t]{11}{*}{ BP } & \multirow{11}{*}{$\begin{array}{l}\text { ribonucleoprotein } \\
\text { complex } \\
\text { biogenesis }\end{array}$} & \multirow[t]{11}{*}{2.039} & \multirow[t]{11}{*}{0.001} & ATXN2;ATXN2L;BCCIP;BRIX1;BYSL;CD2BP2;CELF5;CIRBP;DDX23;DDX27;DDX49;DDX56; \\
\hline & & & & DENR;DHX37;DKC1;EBNA1BP2;EIF3B;EIF3CL;EIF3G;EIF3l;EIF3K;EIF3M;EIF4H;ERI3;EXOSC1; \\
\hline & & & & EXOSC3;EXOSC5;FBL;FRG1;GAR1;GEMIN7;GNL2;GTF3A;GTPBP10;ISG20;KRI1;KRR1;LAS1L; \\
\hline & & & & LSM14A;LSM4;LUC7L2;LYAR;MCTS1;MPV17L2;MRPL11;MRPL20;MRPL22;MRPS11;MRPS2; \\
\hline & & & & MRTO4;MYBBP1A;NGDN;NHP2;NIP7;NOB1;NOC2L;NOC4L;NOL12;NOL6;NOL8;NOM1;NOP10; \\
\hline & & & & NOP16;NOP2;NOP56;NPM1;NSA2;NSUN5;NVL;PA2G4;PIH1D1;POP4;PPAN;PPAN-P2RY11;PRMT7; \\
\hline & & & & PRPF31;PRPF6;RAN;RBM22;RBMX;RIOK2;RPF2;RPL10;RPL10A;RPL11;RPL12;RPL13A;RPL24;RPL26; \\
\hline & & & & RPL26L1;RPL35;RPL35A;RPL38;RPL5;RPL6;RPL7A;RPLP0;RPS10;RPS14;RPS15;RPS16;RPS17;RPS19; \\
\hline & & & & RPS2;RPS21;RPS23;RPS27;RPS28;RPS5;RPS6;RPS7;RPS8;RPS9;RRP1;RRP9;RSL1D1;RSL24D1; \\
\hline & & & & RUVBL2;SART1;SBDS;SF3A2;SNRPB;SNRPC;SNRPD2;SNRPD3;SNRPF;SNRPG;SRPK2;STRAP; \\
\hline & & & & STYXL1;SURF6;SUV39H1;TAF9;TARBP2;TBL3;TRAF7;TXNL4A;UTP14A;UTP15;UTP20;WDR36;WDR46;XAB2 \\
\hline \multirow[t]{8}{*}{ BP } & \multirow{8}{*}{$\begin{array}{l}\text { translational } \\
\text { initiation }\end{array}$} & \multirow[t]{8}{*}{2.207} & \multirow[t]{8}{*}{0.000} & ATF4;DENR;EIF1;EIF1AY;EIF2AK1;EIF2B1;EIF3B;EIF3CL;EIF3G;EIF3I;EIF3K;EIF3M;EIF4B; \\
\hline & & & & EIF4E1B;EIF4EBP1;EIF4H;EIF6;HSPB1;LARP1;MCTS1;MTFMT;MTIF3;NPM1;PABPC1;PAIP1; \\
\hline & & & & PAIP2;PPP1R15A;RPL10;RPL10A;RPL11;RPL12;RPL13;RPL13A;RPL14;RPL17;RPL18;RPL18A; \\
\hline & & & & RPL21;RPL22;RPL24;RPL26;RPL28;RPL29;RPL3;RPL30;RPL31;RPL32;RPL34;RPL35;RPL35A; \\
\hline & & & & RPL36;RPL36A;RPL37;RPL37A;RPL38;RPL39;RPL4;RPL41;RPL5;RPL6;RPL7A;RPL8;RPL9;RPLP0; \\
\hline & & & & RPLP1;RPLP2;RPS10;RPS11;RPS13;RPS14;RPS15;RPS15A;RPS16;RPS17;RPS18;RPS19;RPS2; \\
\hline & & & & RPS20;RPS21;RPS23;RPS24;RPS26;RPS27;RPS28;RPS29;RPS3;RPS4X;RPS4Y1;RPS5;RPS6;RPS7; \\
\hline & & & & RPS8;RPS9;RPSA;RXRA;UBA52;YTHDF1 \\
\hline \multirow[t]{9}{*}{$\mathrm{CC} / \mathrm{MF}$} & \multirow[t]{9}{*}{ ribosome } & \multirow[t]{9}{*}{2.306} & \multirow[t]{9}{*}{0.000} & APEX1;AURKAIP1;C12orf65;CHCHD1;DENR;EEF2;GADD45GIP1;HSPA14;LARP4;MCTS1;MPV17L2; \\
\hline & & & & MRPL11;MRPL14;MRPL20;MRPL21;MRPL22;MRPL27;MRPL3;MRPL32;MRPL34;MRPL36;MRPL4; \\
\hline & & & & MRPL41;MRPL42;MRPL47;MRPL51;MRPL52;MRPL54;MRPS11;MRPS12;MRPS15;MRPS17;MRPS2; \\
\hline & & & & MRPS21;MRPS24;MRPS26;MRPS33;MRPS34;MRPS36;MT3;MTG1;NAA10;NDUFA7;NDUFAB1;RBM3; \\
\hline & & & & RPL10;RPL10A;RPL11;RPL12;RPL13;RPL13A;RPL17;RPL18;RPL18A;RPL21;RPL24;RPL26;RPL26L1; \\
\hline & & & & RPL28;RPL29;RPL3;RPL30;RPL31;RPL32;RPL34;RPL35;RPL35A;RPL36;RPL36AL;RPL37;RPL37A; \\
\hline & & & & RPL38;RPL39;RPL4;RPL41;RPL5;RPL6;RPL7A;RPL8;RPL9;RPLP0;RPLP1;RPLP2;RPS10;RPS11; \\
\hline & & & & RPS13;RPS14;RPS15;RPS15A;RPS16;RPS17;RPS18;RPS19;RPS2;RPS21;RPS23;RPS26;RPS27; \\
\hline & & & & RPS28;RPS29;RPS3;RPS4X;RPS4Y1;RPS4Y2;RPS5;RPS6;RPS6KL1;RPS7;RPS8;RPS9;RRBP1;RSL24D1; \\
\hline
\end{tabular}
discovery rate. 


\begin{tabular}{|c|c|c|c|}
\hline Gene set & NES & FDR & Gene (mRNA) symbols \\
\hline \multirow[t]{7}{*}{ KEGG ribosome } & 2.620 & 0.000 & FAU;MRPL11;MRPL14;MRPL20;MRPL21;MRPL22;MRPL27;MRPL3;MRPL32;MRPL34;MRPL36; \\
\hline & & & MRPL4;MRPS11;MRPS12;MRPS15;MRPS17;MRPS2;MRPS21;RPL10;RPL10A;RPL11; \\
\hline & & & RPL12;RPL13;RPL13A;RPL17;RPL18;RPL18A;RPL21;RPL24;RPL26;RPL26L1;RPL28;RPL29; \\
\hline & & & RPL3;RPL30;RPL31;RPL32;RPL34;RPL35;RPL35A;RPL36;RPL36AL;RPL37;RPL37A;RPL38;RPL39; \\
\hline & & & RPL4;RPL41;RPL5;RPL6;RPL7A;RPL8;RPL9;RPLP0;RPLP1;RPLP2;RPS10;RPS11;RPS13;RPS14; \\
\hline & & & RPS15;RPS15A;RPS16;RPS17;RPS18;RPS19;RPS2;RPS21;RPS23;RPS26;RPS27;RPS28;RPS29; \\
\hline & & & RPS3;RPS4X;RPS4Y1;RPS4Y2;RPS5;RPS6;RPS7;RPS8;RPS9;RSL24D1;UBA52 \\
\hline
\end{tabular}

BP, biological process; CC, cellular component; MF, molecular function; KEGG, Kyoto Encyclopedia of Genes and Genomes; NES, normalized enrichment score discovery rate.

In summary, higher expression of NSUN5 was positively correlated with translation-related mRNA, BPs, CCs, MFs and KEGG pathways in human cancers.

\section{Discussion}

In the present study, by data mining, we found that out of all the eight kinds of RCMTs, six kinds of RCMTs (NSUN1, NSUN2, NSUN3, NSUN4, NSUN5 and TRDMT1) were generally upregulated in human cancers. It indicates that $\mathrm{m}^{5} \mathrm{C}$ and RCMTs may play important roles widely in human cancers. In fact, it has been reported that in leukemia, NSUN3 and TRDMT1 could bind hnRNPK, NSUN1 could bind BRD4, both leading to formation of 5-Azacitidine-sensitive chromatin structure. ${ }^{[6]}$ While NSUN2 could drive bladder cancer progression by stabilizing the mRNA of HDGF through upregulating its $\mathrm{m}^{5} \mathrm{C}$ level. ${ }^{[7]}$ However, the function of $\mathrm{m}^{5} \mathrm{C}$ and RCMTs and its mechanism in other human cancers remain largely unclear. Researches on these aspects should be carried on in the future.

It has been reported that NSUN5 is a rRNA methyltransferase, introducing $\mathrm{m}^{5} \mathrm{C} 3782$ into human $28 \mathrm{~S}$ rRNA. ${ }^{[11]}$ NSUN5 deficiency altered ribosome function, leading to impaired global protein synthesis and normal growth. ${ }^{[11]}$ In this work, we revealed that NSUN5 was the most obviously upregulated RCMT, that higher expression of NSUN5 predicted poorer prognosis, and that NSUN5 expression was positively correlated with translation-related mRNAs, BPs, CCs, MFs and KEGG pathways in multiple human cancers. In addition, the hypomethylation of NSUN5 promoter may be responsible for its upregulation in cancers.

To sum up, we assumed that higher expression of NSUN5 could promote growth by inducing global translation through upregulating translation-related mRNAs in human cancers. The role of NSUN5 in human cancers should be confirmed by biological experiments and the mechanism how NSUN5 upregulates translation-related mRNAs needs further exploration.

\section{Conclusions}

Totally, bioinformatics analysis showed that NSUN5 was an oncogene, overexpressed NSUN5 predicted poorer prognosis and was positively correlated with translation in human cancers.

\section{Methods GEPIA2}

The "Single Gene Analysis-Boxplots" module in GEPIA2 ${ }^{[8]}$ (http://gepia2.cancer-pku.cn/\#index) was used to compare the expression of each RCMT one by one in human cancers and that in associated normal tissues. The cancer data were from TCGA (https://www.cancer.gov/aboutnci/organization/ccg/research/structural-genomics/tcga) cancer data. The normal tissues data were from TCGA normal data and GTEx (https://commonfund.nih.gov/GTEx/) data.

The "Single Gene Analysis-Survival Analysis" module in GEPIA2 was used to analyze the prognostic value of NSUN5 in human cancers. The median expression of NSUN5 was used as the cutoff, which distinguished High-NSUN5-Group from Low-NSUN5-Group.

\section{UALCAN}

The "TCGA analysis" module in UALCAN ${ }^{[9]}$ (http://ualcan.path.uab.edu/) was used to compare the promoter methylation level of NSUN5 in human cancers and that in associated normal tissues. DNA methylation level was indicated by Beta value ranging from 0 (unmethylated) to 1 (fully methylated).

\section{LinkedOmics}

The "LinkFinder" module in LinkedOmics[10] (http://linkedomics.org/login.php) was used to find NSUN5-correlated genes each cancer one by one. Both "Search Dataset" and "Target Dataset" were chosen as "RNAseq", and the statistical method was chosen as "Spearman Correlation test". 
NSUN5-correlated genes ("Association Result") was then used to perform enrichment analysis in the "LinkInterpreter-GSEA (Gene Set Enrichment Analysis)" module in LinkedOmics. Biological process, Cellular Component, Molecular Function and KEGG pathway analyses were performed one by one. The "Rank Criteria" was chosen as "Statistic" ("spearman correlation" in this situation), the "Minimum Number of Genes" was chosen as "3" and the "Simulations" was chosen as " 500 ".

\section{Abbreviations}

ACC, Adrenocortical carcinoma; BLCA, Bladder Urothelial Carcinoma; BP, biological process; BRCA, Breast invasive carcinoma; CC, cellular component; CESC, Cervical squamous cell carcinoma and endocervical adenocarcinoma; CHOL, Cholangiocarcinoma; COAD, Colon adenocarcinoma; DLBC, Lymphoid Neoplasm Diffuse Large B-cell Lymphoma; ESCA, Esophageal carcinoma; FDR, false discovery rate; GBM, Glioblastoma multiforme; GO, gene ontology; HNSC, Head and Neck squamous cell carcinoma ; KEGG, Kyoto Encyclopedia of Genes and Genomes; KICH, Kidney Chromophobe; KIRC, Kidney renal clear cell carcinoma; KIRP, Kidney renal papillary cell carcinoma ; LAML, Acute Myeloid Leukemia; LGG, Brain Lower Grade Glioma; LIHC, Liver hepatocellular carcinoma; LUAD, Lung adenocarcinoma; LUSC, Lung squamous cell carcinoma; m5C, 5-methylcytosine; m6A, N6-methyladenosine; MF, molecular function; mRNA, messenger RNA; OS, overall survival; OV, Ovarian serous cystadenocarcinoma; PAAD, Pancreatic adenocarcinoma; PCPG, Pheochromocytoma and Paraganglioma; PRAD, Prostate adenocarcinoma; RCMT, RNA m5C methyltransferase; READ, Rectum adenocarcinoma; RFS, recurrence free survival; rRNA, ribosomal RNA; SARC, Sarcoma; SKCM, Skin Cutaneous Melanoma; STAD, Stomach adenocarcinoma; TGCT, Testicular Germ Cell Tumors; THCA, Thyroid carcinoma; THYM, Thymoma; tRNA, transfer RNA; UCEC, Uterine Corpus Endometrial Carcinoma; UCS, Uterine Carcinosarcoma; UVM, Uveal Melanoma.

\section{Declarations}

\section{Ethics approval and consent to participate:}

Not applicable.

\section{Consent for publication:}

Not applicable.

\section{Competing interests:}

The authors declare that they have no competing interests.

\section{Funding:}

This work was supported by Chinese Medicine Inheritance and Innovation "100 Million" Talent Project (Qi Huang Scholar) and Three-year Action Plan Project of Shanghai Traditional Chinese Medicine Development\ZY(2018-2020)-CCCX-2004-01区.

\section{Authors' contributions:}

Xiao-wen Zhang: Methodology, Formal analysis and Writing-Original Draft;

Yan Huang: Methodology, Validation and Writing - Review \& Editing;

Lu-yi Wu: Resources, Data Curation and Writing-Original Draft;

Qin Qi: Methodology;

Rui Zhong: Resources;

Shan-shan Li: Visualization;

Ji-meng Zhao: Software;

Hui-rong Liu: Conceptualization, Supervision and Funding acquisition;

Huan-gan Wu: Conceptualization, Project administration and Funding acquisition.

\section{Acknowledgements:}

We appreciated Ya-nan Liu (Yueyang Clinical Medical College, Shanghai University of Traditional Chinese Medicine, Shanghai, China) for editing the manuscript. 


\section{Availability of data and materials:}

All data generated or analyzed during this study are included in this published article and its supplementary information files.

\section{References}

1. BOCCALETTO P, MACHNICKA M A PURTAE, et al. MODOMICS: a database of RNA modification pathways. 2017 update [J]. Nucleic acids research. 2018;46(D1):D303-d7.

2. CHEN X Y, ZHANG J, ZHU JS. The role of m(6)A RNA methylation in human cancer [J]. Mol Cancer. 2019;18(1):103.

3. GARCIA-VILCHEZ R, SEVILLA A. BLANCO S. Post-transcriptional regulation by cytosine-5 methylation of RNA [J]. Biochimica et biophysica acta Gene regulatory mechanisms, 2019, 1862(3): 240-52.

4. HUANG T, CHEN W, LIU J, et al. Genome-wide identification of mRNA 5-methylcytosine in mammals [J]. Nat Struct Mol Biol. 2019;26(5):380-8.

5. BOHNSACK K E, HOBARTNER C. BOHNSACK M T. Eukaryotic 5-methylcytosine (m(5)C) RNA Methyltransferases: Mechanisms, Cellular Functions, and Links to Disease [J]. Genes. 2019;10(2):1.

6. CHENG JX, CHEN L, LI Y, et al. RNA cytosine methylation and methyltransferases mediate chromatin organization and 5-azacytidine response and resistance in leukaemia [J]. Nature communications. 2018;9(1):1163.

7. CHEN X, LI A, SUN B F, et al. 5-methylcytosine promotes pathogenesis of bladder cancer through stabilizing mRNAs [J]. Nat Cell Biol. 2019;21(8):978-90.

8. TANG Z, KANG B, LI C, et al. GEPIA2: an enhanced web server for large-scale expression profiling and interactive analysis [J]. Nucleic acids research, 2019, 47(W1): W556-w60.

9. CHANDRASHEKAR DS, BASHEL B, BALASUBRAMANYA S A H, et al. UALCAN: A Portal for Facilitating Tumor Subgroup Gene Expression and Survival Analyses [J]. Neoplasia (New York, NY), 2017, 19(8): 649 - 58.

10. VASAIKAR SV, STRAUB P, WANG J, et al. LinkedOmics: analyzing multi-omics data within and across 32 cancer types [J]. Nucleic acids research, 2018 , 46(D1): D956-d63.

11. HEISSENBERGER C, LIENDL L, NAGELREITER F, et al. Loss of the ribosomal RNA methyltransferase NSUN5 impairs global protein synthesis and normal growth [J]. Nucleic acids research. 2019;47(22):11807-25.

\section{Figures}



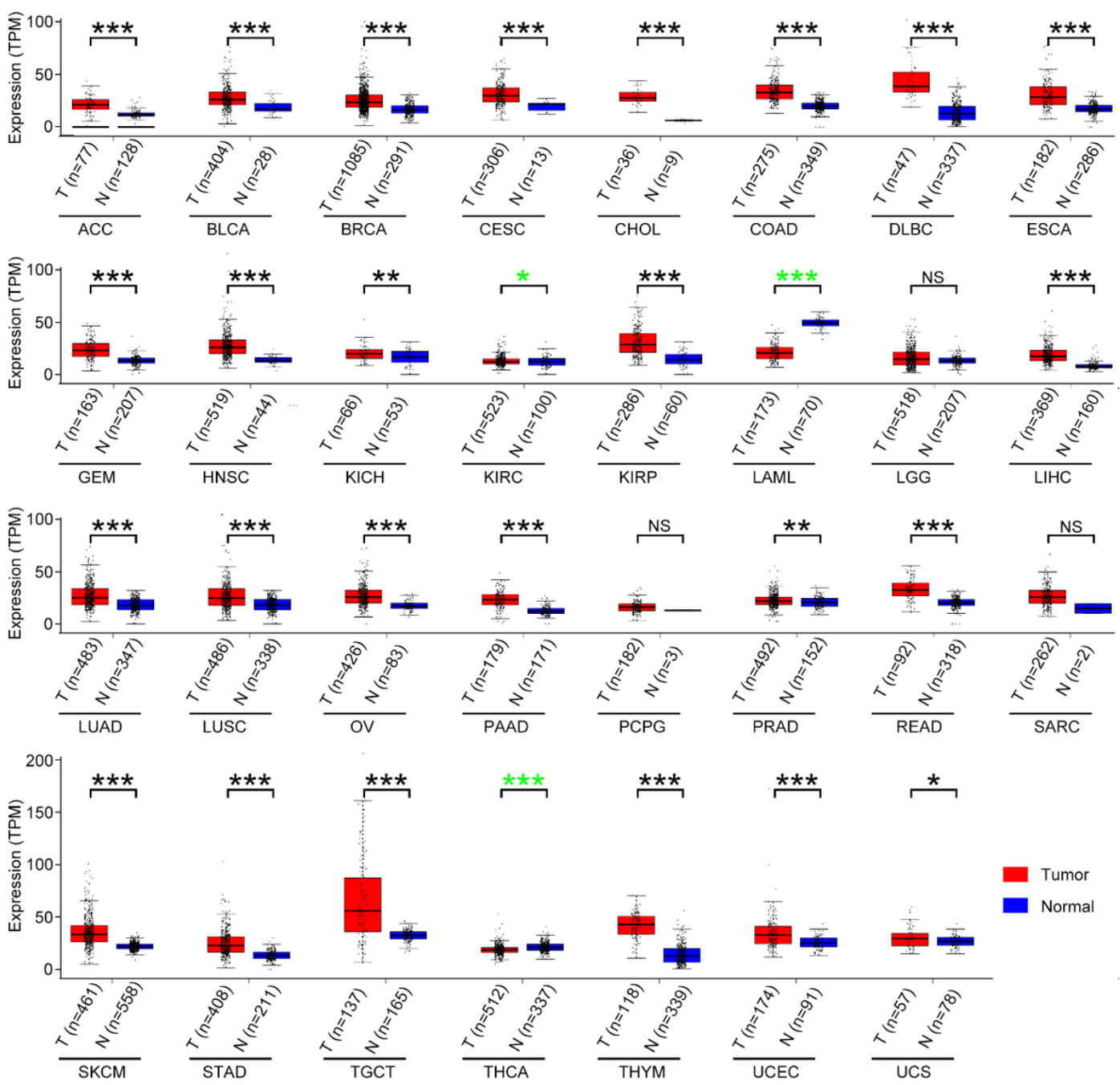

Figure 1

The expression of NSUN5 in 31 types of cancers and associated tissues (GEPIA2). ${ }^{*}, \mathrm{P}<0.05 ; * \star, P<0.01 ; * \star *, P<0.001$; black and green asterisk indicate upregulation and downregulation in tumor, respectively. NS, not significant. TPM, transcripts per kilobase of exon model per million mapped reads. 

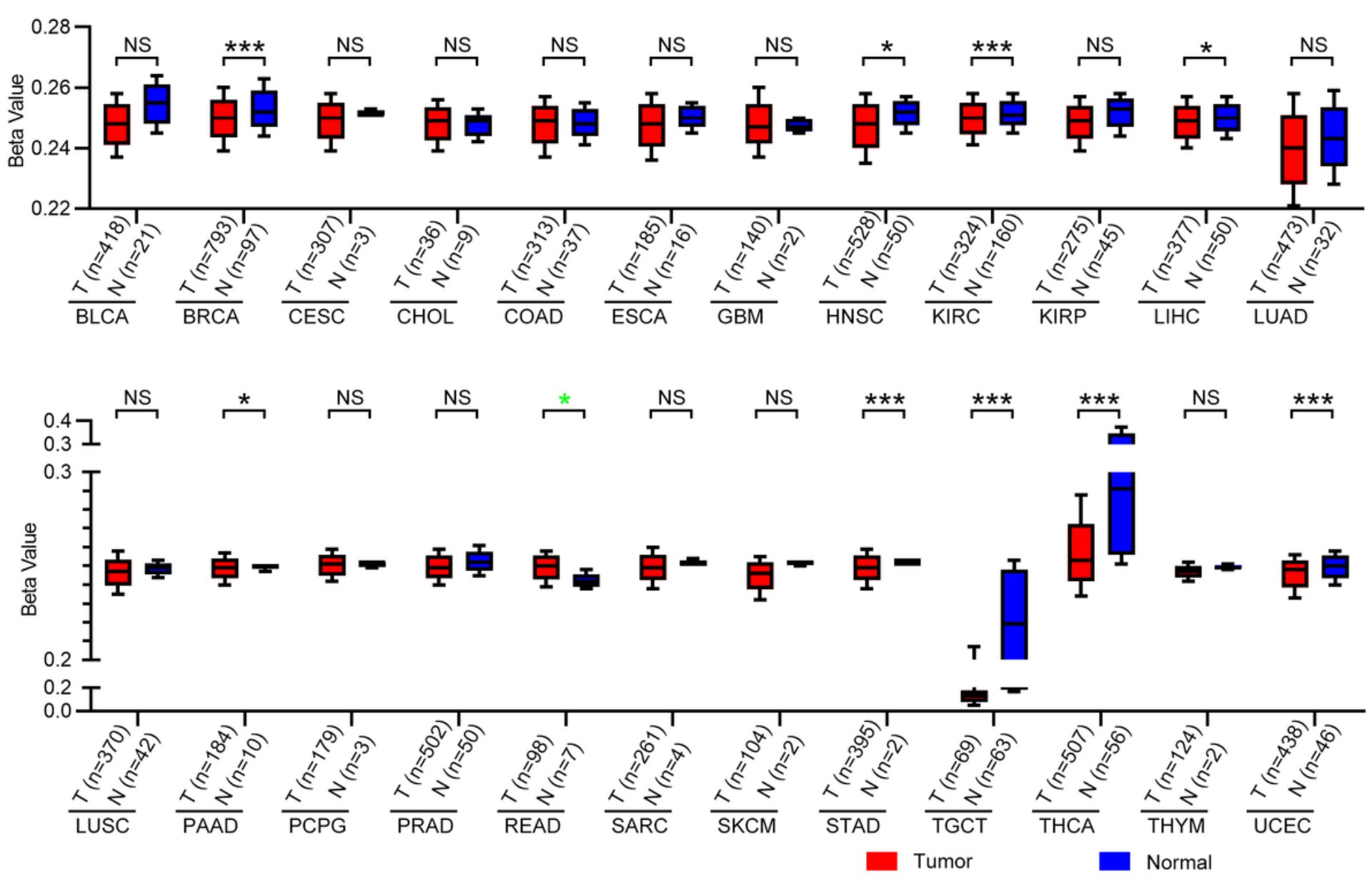

Figure 2

The promoter methylation level of NSUN5 in 28 types of cancers and associated normal tissues. (UALCAN) *, P<0.05; **, P<0.01; ***, P<0.001; black and green asterisk indicate upregulation and downregulation in tumor, respectively. NS, not significant. 

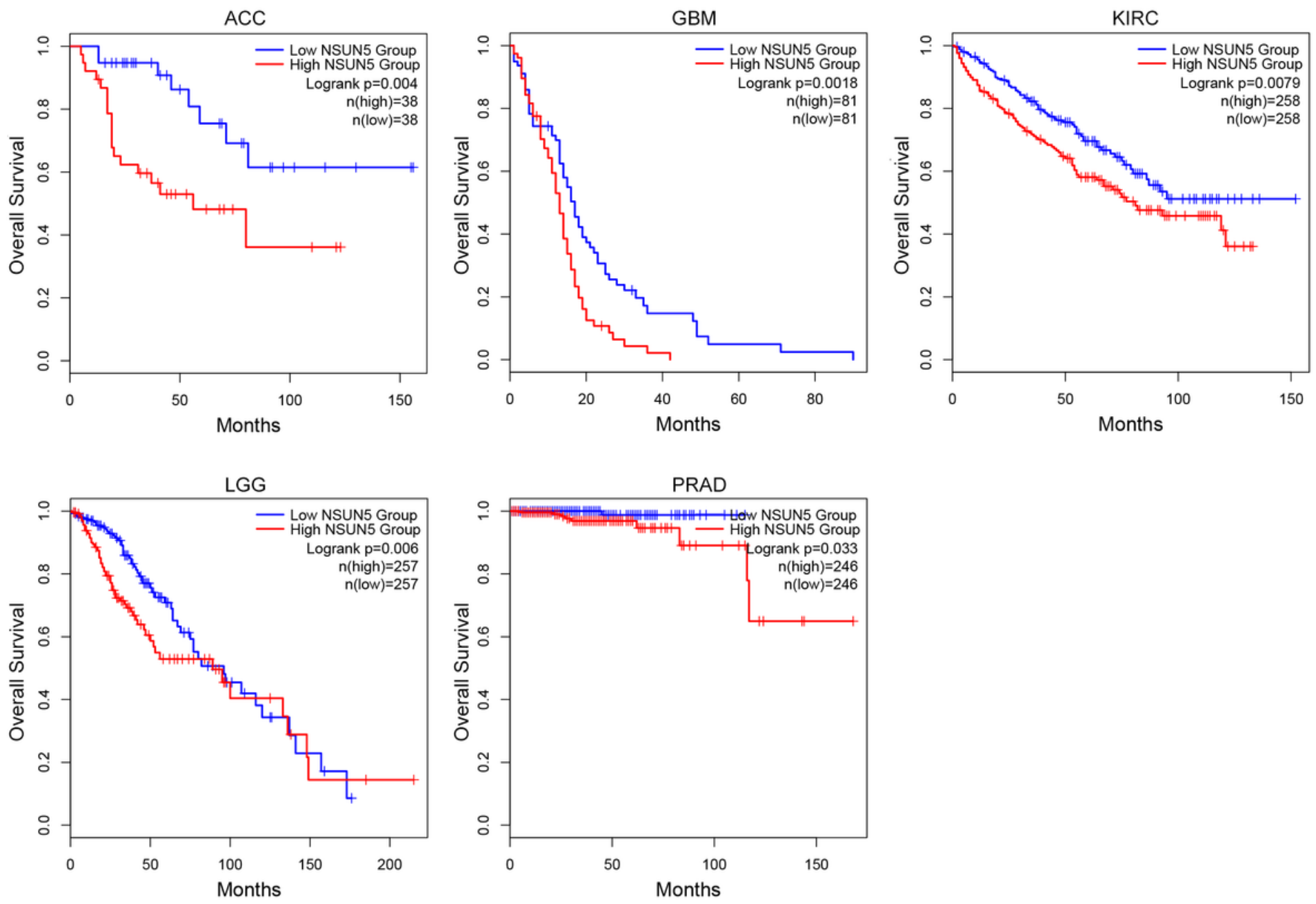

\section{Figure 3}

Higher expression of NSUN5 predicts lower overall survival for ACC, GBM, KIRC, LGG and PRAD patients. (GEPIA2) 
ACC

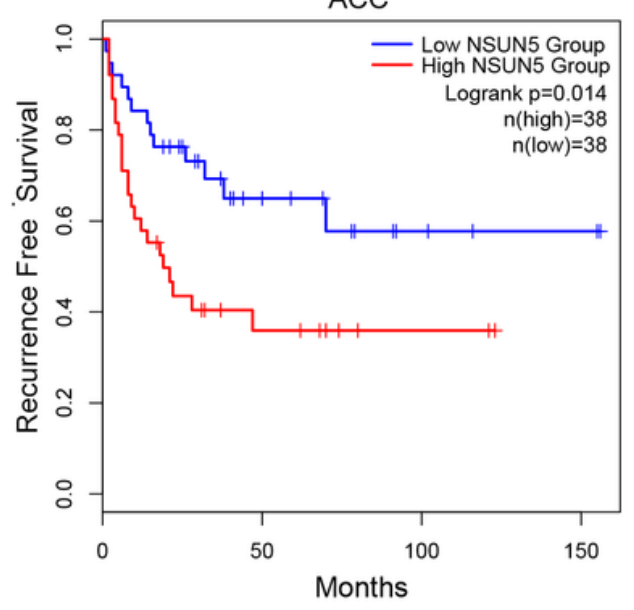

LUSC

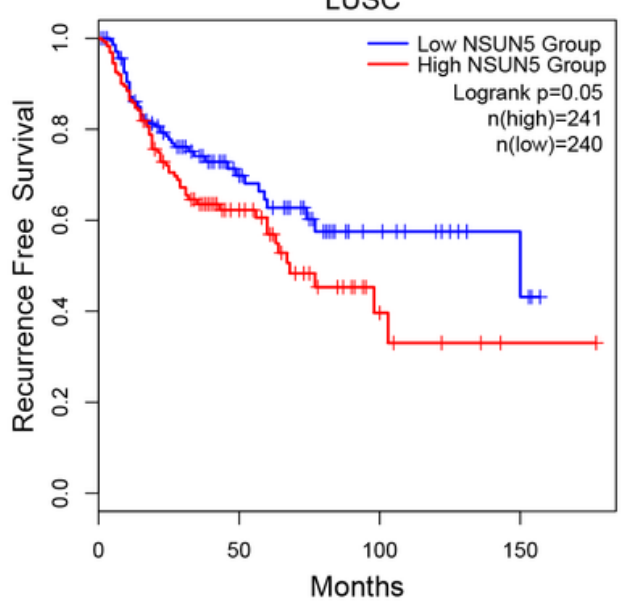

LGG

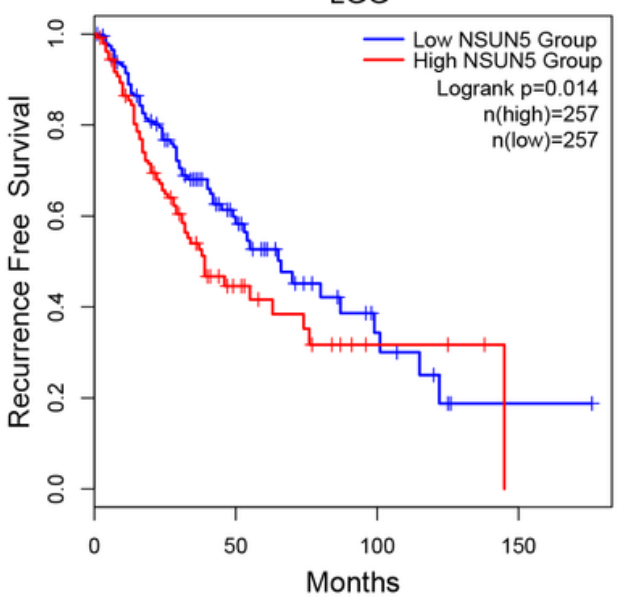

PRAD

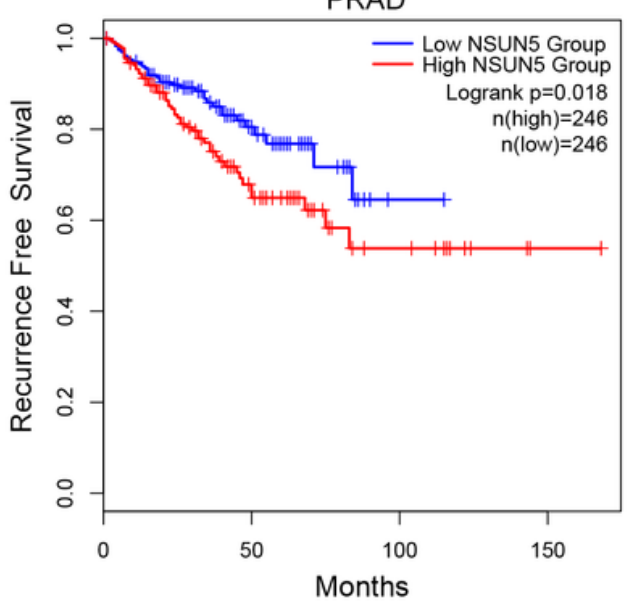

LIHC

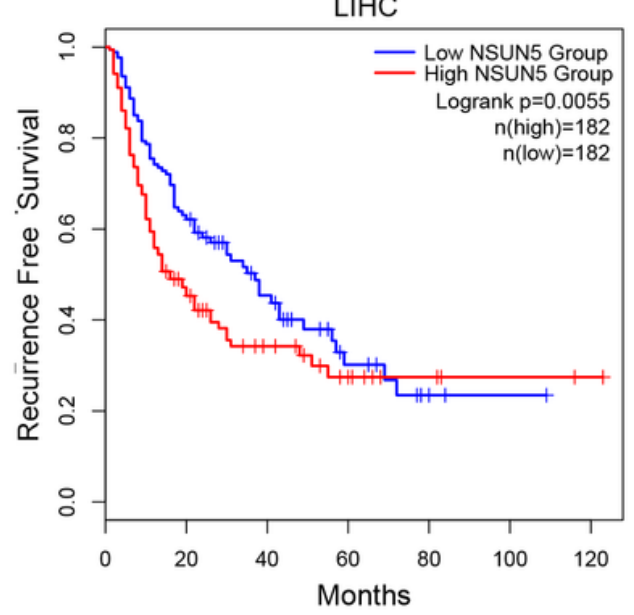

\section{Figure 4}

Higher expression of NSUN5 predicts lower recurrence free survival for ACC, LGG, LIHC, LUSC and PRAD patients. (GEPIA2) 
A
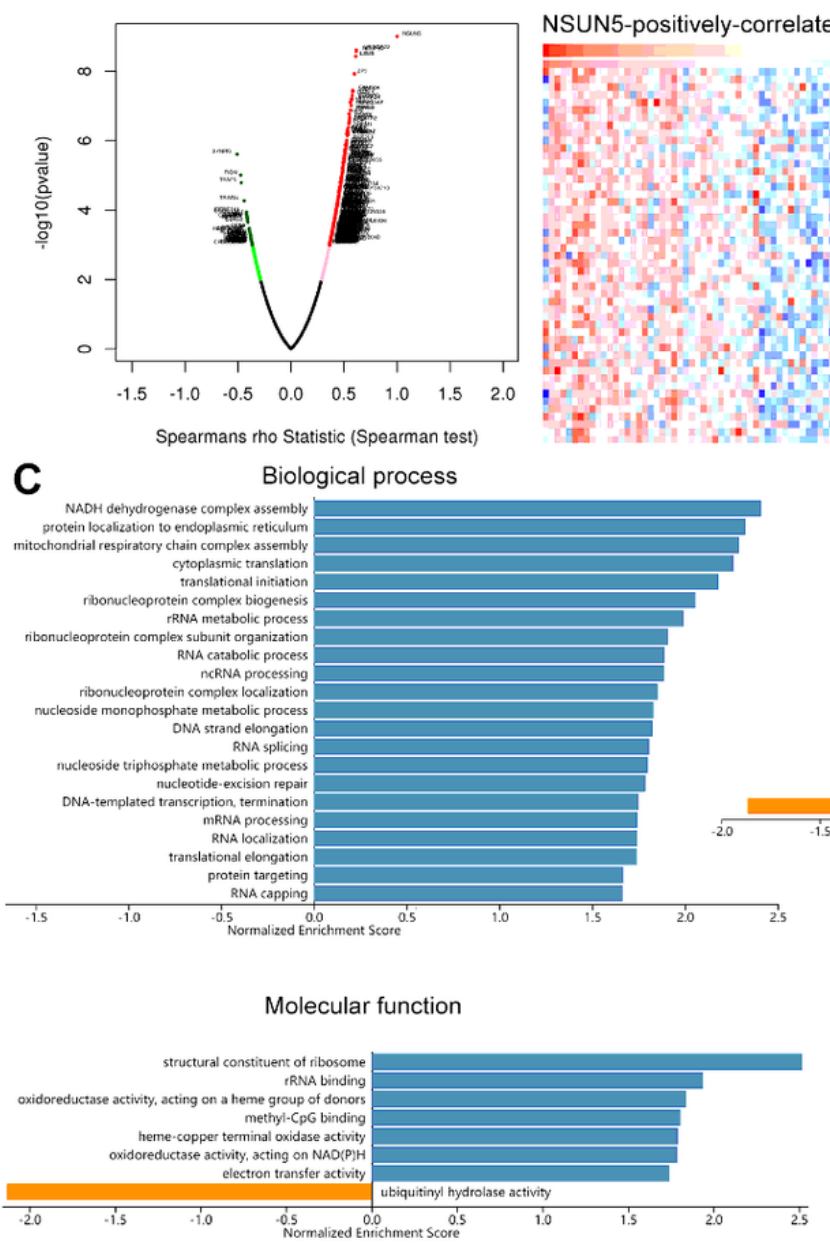

B
NSUN5-positively-correlated genes (top 50) NSUN5-negatively-correlated genes (top 50)
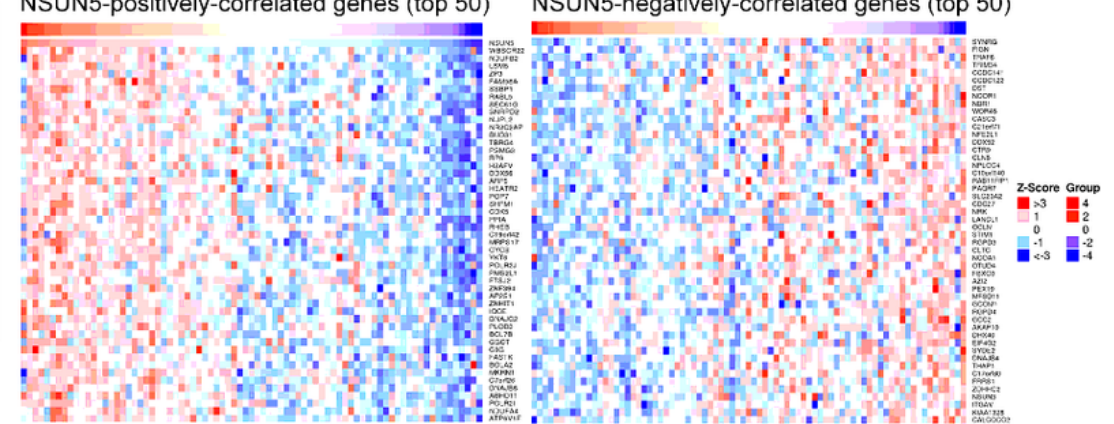

Cellular component

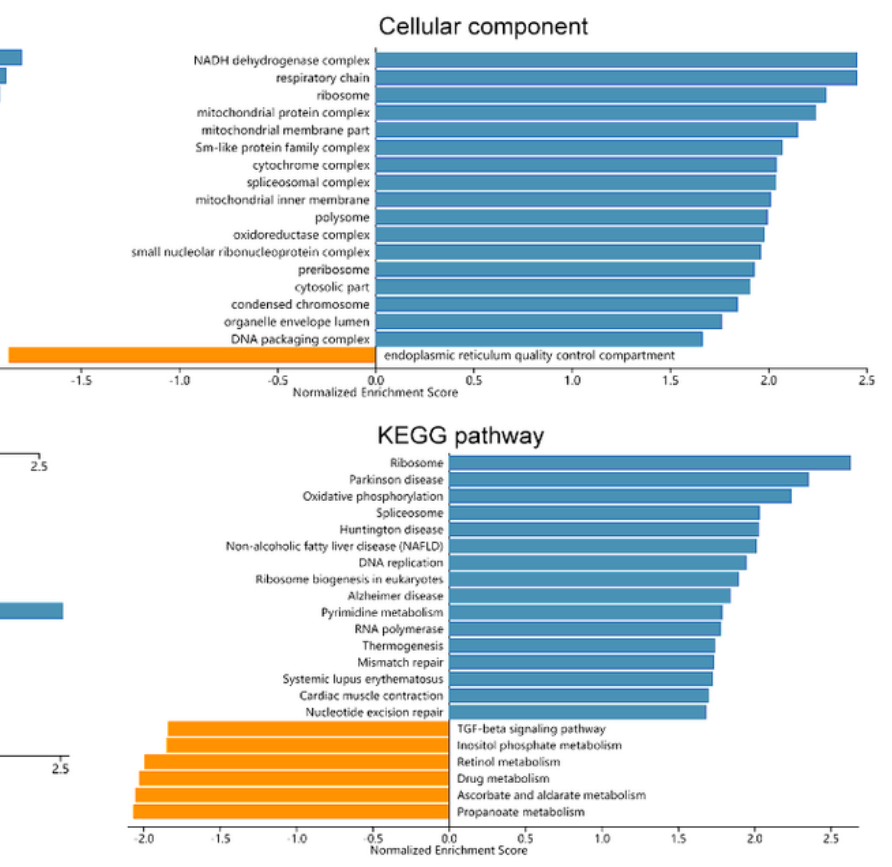

Figure 5

The BPs (biological processes), CCs (cellular components), MFs (molecular functions) and KEGG pathways analyses of NSUN5-correlated genes in ACC. (LinkedOmics)

\section{Supplementary Files}

This is a list of supplementary files associated with this preprint. Click to download.

- SupplementaryTables113.xlsx 